\title{
Risk Arbitrage and Hedging to Acceptability under Transaction Costs
}

\author{
Emmanuel Lepinette · Ilya Molchanov
}

April 16, 2020

\begin{abstract}
The classical discrete time model of proportional transaction costs relies on the assumption that a feasible portfolio process has solvent increments at each step. We extend this setting in two directions, allowing for convex transaction costs and assuming that increments of the portfolio process belong to the sum of a solvency set and a family of multivariate acceptable positions, e.g. with respect to a dynamic risk measure. We describe the sets of superhedging prices, formulate several no (risk) arbitrage conditions and explore connections between them. In the special case when multivariate positions are converted into a single fixed asset, our framework turns into the no good deals setting. However, in general, the possibilities of assessing the risk with respect to any asset or a basket of the assets lead to a decrease of superhedging prices and the no arbitrage conditions become stronger. The mathematical technique relies on results for unbounded and possibly non-closed random sets in Euclidean space.
\end{abstract}

Keywords acceptance set · risk arbitrage · risk measure - superhedging · good deal $\cdot$ solvency set $\cdot$ random set $\cdot$ transaction costs

Mathematics Subject Classification (2010) 91G20, 60D05, 60G42

E. Lepinette

Paris-Dauphine University, Place du Maréchal De Lattre De Tassigny, 75775 Paris cedex 16, France, and GOSAEF, Tunis-El Manar University, 2092-ElManar, Tunisia

E-mail: emmanuel.lepinette@ceremade.dauphine.fr

I. Molchanov

Institute of Mathematical Statistics and Actuarial Science, University of Bern, Alpeneggstr. 22, 3012 Bern, Switzerland

E-mail: ilya.molchanov@stat.unibe.ch 


\section{Introduction}

Transaction costs in financial markets are often described using solvency sets, which consist of all financial positions (in physical quantities) regarded better than the zero position or at least equivalent to it. In the dynamic discrete time setting, the solvency sets form a set-valued random process $\left(K_{t}\right)_{t=0, \ldots, T}$ adapted to the underlying filtration $\left(\mathcal{F}_{t}\right)_{t=0, \ldots, T}$. The no arbitrage conditions are usually formulated in terms of selections of these solvency sets, that is, for random vectors that a.s. belong to the solvency sets and so correspond to particular choices of solvent portfolios. In many cases, solvency sets are polyhedral cones and the corresponding model is known as Kabanov's model with proportional transaction costs, see [23,24,32, where the no arbitrage conditions are thoroughly discussed.

If $\xi$ is a claim that matures at time $T$, then the set of initial positions suitable as a starting value for a self-financing portfolio process $\left(V_{t}\right)_{t=0, \ldots, T}$ paying $\xi$ at maturity forms the family of superhedging prices for $\xi$. In the multivariate setting, the starting values are vectors which are not necessarily comparable to each other, and so, instead of comparing them by a single numerical quantity, it is sensible to look at the whole set of superhedging prices. The self-financing requirement amounts to the fact that the increment $V_{t-1}-V_{t}$ of the portfolio process is solvent at all times, that is, it a.s. belongs to $K_{t}$ for all $t$ (in other words, the increment is a selection of $K_{t}$ ).

In order to reduce these superhedging prices, it is possible to require that the shortfall of the terminal value of the portfolio, in comparison with the claim, is acceptable with respect to a certain risk measure. This approach may provide arbitrage opportunities as Good Deals, i.e. terminal claims attainable from the zero capital and such that the risk of the claim is strictly negative, equivalently, the utility is strictly positive. The No Good Deal condition, first introduced in [11] and then formalised in [4,10, requires that this situation is impossible. Unlike the univariate setting, the existence of a good deal in the multivariate setting does not necessarily mean the existence of a claim whose multivariate utility belongs to $\mathbb{R}_{+}^{d}$. Indeed, a vector-valued financial position may be acceptable if some acceptable components compensate for the nonacceptable ones. This may result in various types of arbitrage opportunities.

Indeed, it is possible to strengthen the no arbitrage requirement by also considering hedging strategies, where the self-financing condition is replaced by the acceptability of all intermediate portfolio changes with respect to a dynamic risk measure, see [7. The setting of [7,8 involves at least two assets exchangeable without transaction costs and pinpoints a particular asset that is used as the cash equivalent. The portfolio is converted to its cash equivalent, with the acceptability condition imposed on the increments of these cash values for consecutive time moments. The idea of converting portfolios to a single numerical quantity with acceptable increments in view of superhedging onedimensional claims has been further explored in [5].

However, if there are several currencies (exchangeable with random frictionless rates or with transaction costs), it may well be the case that the 
position expressed in one currency is acceptable, while the position in the other one is not, see [29, Ex. 1.1]. This may lead to regulatory arbitrages, see 34. If the regulator is prepared to apply a relaxed acceptability criterion for one currency, then it would be logical to expect the same policy with respect to another currency or a basket of currencies. We show how to handle this in a way that treats all components of a portfolio in the same manner.

The key idea of this work is to extend the family of self-financing portfolio processes by requiring that $V_{t-1}-V_{t}$ equals the sum of a selection of $K_{t}$ (a solvent position) and another random vector that is not necessarily solvent, but is acceptable with respect to a dynamic multivariate risk measure. It is worth mentioning that $K_{t}$ is only supposed to be convex, contrarily to the classical literature of linear transaction costs. With this hedging to acceptability approach, all components of the portfolio are treated in the same way. Then, $\left(V_{t}\right)_{t=0, \ldots, T}$ is called an acceptable portfolio process. For example, the classical superhedging setting arises if the componentwise conditional essential infimum is chosen as the risk measure, so that acceptable random vectors necessarily have all a.s. non-negative components. The hedging to acceptability substantially increases the choice of possible hedging strategies, but in some cases may lead to arbitrage.

Example 1 Let $r$ be any coherent risk measure. Consider the one period zero interest model with two currencies as the assets. Assume that the exchange rate $\pi$ (so that $\pi$ units of the second asset buy one unit of the first one) at time one is log-normally distributed (in the real world) and the exchanges are free from transaction cost. Then, the positions $\gamma^{\prime}=(-a, \pi a)$ and $\gamma^{\prime \prime}=(a,-\pi a)$ for $a>0$ are reachable from $(0,0)$ at zero cost. Their risks are $(a, \operatorname{ar}(\pi))$ and $(-a, \operatorname{ar}(-\pi))$. In order to secure the capital reserves for $\gamma^{\prime}$, the agent has to reserve $a$ of the first currency and $\operatorname{ar}(\pi)$ of the second one (note that $r(\pi)<0$ ). If the exchange rate at time zero is $\pi_{0}$, the initial cost expressed in the second currency is

$$
\pi_{0} a+\operatorname{ar}(\pi)=a\left(\pi_{0}+r(\pi)\right) .
$$

In order to secure $\gamma^{\prime \prime}$, the initial cost is $a\left(-\pi_{0}+r(-\pi)\right)$. If $\pi_{0}$ does not belong to the interval $[-r(\pi), r(-\pi)]$, then either $\pi_{0}+r(\pi)<0$ or $-\pi_{0}+r(-\pi)<0$, and we let $a$ grow to release infinite capital at time zero. Note that this model does not admit financial arbitrage, since there exists a martingale measure. This example can be easily modified by accounting for proportional transaction costs.

It is recognised by now that risks of multivariate positions involving possible exchanges of assets and transaction costs are described as sets, see [2, 19]. The multiasset setting naturally makes it possible to offset a risky position using various combinations of assets. In this framework it is also natural to consider the family of all attainable positions as a set-valued portfolio. Treating both arguments and values of a risk measure as random sets leads to law invariant risk measures and makes it possible to iterate the construction, which is essential to handle dynamic risk measures. 
One of the aims of this paper is to introduce a geometric characterisation of superhedging prices. On the way, we suggest a constructive definition of dynamic multivariate risks based on the families of acceptable positions and so extend the existing works on dynamic set-valued risk measures 13, 14 by letting the arguments of risks and their values be sets of random vectors in $\mathbb{R}^{d}$. In many instances, these sets may be interpreted as random (possibly, non-closed) sets. The necessary background on random sets is provided in the appendix. In particular, it is shown that the Minkowski (elementwise) sum of two random closed sets is measurable, no matter if the sum is closed or not. Special attention is devoted to the decomposability and infinite decomposability properties, which are the key concepts suitable to relate families of random vectors to selections of random sets.

We refer to [12] and [17] for the basics of static risk measures and to [1] for a survey of the dynamic $\mathcal{L}^{\infty}$-setting, further extended beyond the $\mathcal{L}^{\infty}$-setting by the module approach worked out in [15, 16.

Static risk measures are usually defined on $\mathcal{L}^{p}(\mathbb{R}, \mathcal{F})$ with $p \in[1, \infty]$. However, in many cases, they are well defined also on larger sets of random variables. For example, $r(\xi)=-\operatorname{ess} \inf \xi$ makes sense for all random variables essentially bounded from below by a constant. Similarly, if $r(\xi)=-\mathrm{E} \xi$, then the acceptance set is defined as the family of $\xi$ such that their positive and negative parts satisfy $\mathrm{E} \xi^{+} \geq \mathrm{E} \xi^{-}>\infty$. The boundedness of $\mathrm{E} \xi^{+}$is not required.

To account for similar effects in relation to multivariate dynamic risk measures, we put forward acceptance sets in place of risk measures. The acceptance sets $\mathcal{C}_{t, s}$ with $t \leq s$ are subsets of the sum of the family of $\mathcal{F}_{s}$-measurable random vectors in $\mathbb{R}^{d}$ that admit generalised conditional $p$ th moment with respect to $\mathcal{F}_{t}$ and the family of all $\mathcal{F}_{s}$-measurable random vectors in $\mathbb{R}_{+}^{d}$. Section 2 introduces basic conditions on the acceptance sets and several optional ones.

The dynamic selection risk measure $\mathrm{R}_{t, s}(\Xi)$ for a family $\Xi \subset \mathcal{L}^{0}\left(\mathbb{R}^{d}, \mathcal{F}_{T}\right)$ is introduced as the closure in probability of $\left(\Xi+\mathcal{C}_{t, s}\right) \cap \mathcal{L}^{0}\left(\mathbb{R}^{d}, \mathcal{F}_{t}\right)$. If $\Xi=$ $\mathcal{L}^{0}\left(X, \mathcal{F}_{T}\right)$ is the family of selections for a random closed set $X$, then $\mathrm{R}_{t, s}(X)=$ $\mathrm{R}_{t, s}(\Xi)$ itself is an $\mathcal{F}_{t}$-measurable random closed set. In comparison with [14], this approach explicitly defines a set-valued dynamic risk measure instead of imposing on it some axiomatic properties. This yields a set-valued risk measure with a set-valued argument that can be naturally iterated in the dynamic framework. The conditional convexity of the acceptance sets yields that

$$
\mathrm{R}_{t, s}(\lambda X+(1-\lambda) Y) \supset \lambda \mathrm{R}_{t, s}(X)+(1-\lambda) \mathrm{R}_{t, s}(Y) \quad \text { a.s. }
$$

for any $\lambda \in \mathcal{L}^{0}\left([0,1], \mathcal{F}_{t}\right)$ and random closed sets $X$ and $Y$, meaning that the risk measure is also conditionally convex. The static case of this construction was considered in [29], where properties of selection risk measures in the coherent case are obtained, some of them easily extendable for the dynamic convex setting. Comparing to 29], we work with solvency sets instead of portfolios available at price zero and also allow the argument of the risk measure to be a rather general family of random vectors. 
The hedging to acceptability relies on a sequence $\left(K_{t}\right)_{t=0, \ldots, T}$ of solvency sets and the acceptance sets $\mathcal{C}_{t, s}$ for $0 \leq t \leq s \leq T$. Note that the solvency sets are not assumed to be conical, since non-conical models naturally appear, e.g. in the order book setting, see 3] and [30. An acceptable portfolio process $\left(V_{t}\right)_{t=0, \ldots, T}$ introduced in Section 3 satisfies $V_{t-1}-V_{t}=k_{t}+\eta_{t}$ for $k_{t} \in \mathcal{L}^{0}\left(K_{t}, \mathcal{F}_{t}\right), \eta_{t} \in \mathcal{C}_{t-1, t}$, and all $t$. In other words, the available assets do suffice to pay for the portfolio at the next step up to an amount acceptable with respect to some risk measure. The strongest acceptability condition assumes that $\mathcal{C}_{t-1, t}$ consists of random vectors with non-negative components and yields the classical arbitrage theory for markets with transaction costs, see 24. The weakest acceptability requirement presumes that all $\eta_{t}$ from $\mathcal{C}_{t-1, t}$ have non-negative $\mathcal{F}_{t-1}$-conditional expectations.

If $\xi$ is a terminal claim on $d$ assets, then $\Xi_{t}^{\xi}$ denotes the set of all initial endowments at time $t$ that ensure the existence of an acceptable portfolio process paying $\xi$ at maturity, that is, $V_{T} \in \xi+K_{T}$ a.s. Equivalently, $\Xi_{t}^{\xi}$ is the family of $\mathcal{F}_{t}$-measurable elements of $\left(\xi-\mathrm{A}_{t, T}\right)$, where $\mathrm{A}_{t, T}$ is the set of claims attainable at time $T$ starting from zero investment at time $t$. The family $\Xi_{t}^{\xi}$ may be used to assess the risk associated with $\xi$ at time $t$.

The no arbitrage conditions we study are imposed on the set of superhedging prices $\Xi_{t}^{0}$ for the zero claim $\xi=0$. They may be reformulated as no arbitrage conditions on the set of attainable claims, which are weaker than the usual ones of the literature. These no risk arbitrage conditions are introduced and analysed in Section 4. In difference to [7, we do not rely on the weak compactness of the duals to the acceptance sets and we do not need to pinpoint any reference asset. It should be noted that the risk arbitrage only makes sense in the multiasset setting with some trading opportunities between the assets; if $K_{t}=\mathbb{R}_{+}^{d}$ (which is always the case on the line), then all no risk arbitrage conditions automatically hold.

It is shown that, in some cases, it is possible to represent the families of capital requirements as a set-valued process, and the no risk arbitrage conditions, for linear transaction costs, can be characterised in terms of weakly consistent price systems, so providing a variant of the Fundamental Theorem of Asset Pricing in our framework, see Theorem 4.4.

A comparison of our approach with the no good deals setting is provided in Section 5. It is shown that our approach imposes stronger no arbitrage conditions that are more difficult to check, but which result in lower superhedging prices.

Note that the sets $\mathcal{C}_{t, s}$ of acceptable positions always contain the family $\mathcal{L}^{0}\left(\mathbb{R}_{+}^{d}, \mathcal{F}_{s}\right)$ of random vectors with a.s. non-negative components and, in many cases, $\mathcal{C}_{t, s}$ is a subset of the family of random vectors with non-negative generalised conditional expectation given $\mathcal{F}_{t}$. Thus, the no risk arbitrage conditions are sandwiched between those for the risk measure based on the conditional essential infimum and on the conditional expectation. The first choice corresponds to the classical financial arbitrage with transaction costs, where our no risk arbitrage conditions become the classical no arbitrage conditions. 
Section 6 recovers and extends several results from [24]. In this classical setting, our approach yields a new geometric interpretation of the sets of superhedging prices with possibly non-conical solvency sets; it is formulated using the concept of the conditional core of a random set elaborated in 26 . The result applies also in some cases when the classical consistent price systems characterisation fails.

In Section 7 we characterise no arbitrage conditions arising by adopting the generalised conditional expectation as the acceptability criterion. These are the strongest no arbitrage conditions in our framework; their validity ensures the absence of arbitrage for all acceptance criteria satisfying a dynamic version of the dilatation monotonicity condition from 9 .

The results from Sections 6 and 7 are illustrated on a two-asset example in Section 8

\section{Dynamic acceptance sets and selection risk measures}

\subsection{Definition and main properties}

Let $\left(\Omega,\left(\mathcal{F}_{t}\right)_{t=0, \ldots, T}, \mathrm{P}\right)$ be a stochastic basis on a complete probability space such that $\mathcal{F}_{0}$ is the trivial $\sigma$-algebra and $\mathcal{F}_{T}=\mathcal{F}$. In the following, we endow random vectors and events with a subscript that indicates the $\sigma$-algebras they are measurable with respect to. The subscript is often omitted for $\mathcal{F}_{T^{-}}$ measurable random vectors.

Let $\mathcal{L}^{p}\left(\mathbb{R}^{d}, \mathcal{F}\right)$ with $p \in[1, \infty]$ be the family of $p$-integrable random vectors (essentially bounded if $p=\infty$ ), and let $\mathcal{L}^{0}\left(\mathbb{R}^{d}, \mathcal{F}\right)$ be the family of all random vectors in $\mathbb{R}^{d}$. The closure in the strong topology in $\mathcal{L}^{p}\left(\mathbb{R}^{d}, \mathcal{F}\right)$ for $p \in[1, \infty)$ is denoted by $\mathrm{cl}_{p}$, and $\mathrm{cl}_{0}$ is the closure in probability in $\mathcal{L}^{0}\left(\mathbb{R}^{d}, \mathcal{F}\right)$. If $p=\infty$, the closure is considered with respect to the a.s. convergence of uniformly bounded sequences.

For a sub- $\sigma$-algebra $\mathcal{H} \subset \mathcal{F}$, denote $\mathcal{L}_{\mathcal{H}}^{p}\left(\mathbb{R}^{d}, \mathcal{F}\right)$ the module of $\mathcal{F}$-measurable random vectors that can be represented as $\gamma \xi$ with $\xi \in \mathcal{L}^{p}\left(\mathbb{R}^{d}, \mathcal{F}\right)$ and $\gamma \in$ $\mathcal{L}^{0}(\mathbb{R}, \mathcal{H})$, see [15, Ex. 2.5]. In particular, $\mathcal{L}_{\mathcal{H}}^{1}\left(\mathbb{R}^{d}, \mathcal{F}\right)$ is the family of all $\xi$ that admit the generalised conditional expectation $\mathrm{E}^{g}(\xi \mid \mathcal{H})$ with respect to $\mathcal{H}$, see [26. Lemma B.3]. Following [15, Ex. 2.5], the module norm is defined by

$$
\|\xi\|_{p, \mathcal{H}}= \begin{cases}\mathrm{E}\left(\|\xi\|^{p} \mid \mathcal{H}\right)^{1 / p}, & p \in[1, \infty), \\ {\operatorname{ess} \sup _{\mathcal{H}}\|\xi\|,} \| p=\infty,\end{cases}
$$

where $\operatorname{ess}_{\sup }\|\xi\|$ is the $\mathcal{H}$-measurable essential supremum of $\|\xi\|$, see [17, Appendix A.5]. We endow the space $\mathcal{L}_{\mathcal{H}}^{p}\left(\mathbb{R}^{d}, \mathcal{F}\right)$ with the topology of $\mathcal{L}_{\mathcal{H}^{-}}^{p}$ convergence by assuming that $\xi^{n}$ converges to $\xi$ if $\left\|\xi^{n}-\xi\right\|_{p, \mathcal{H}} \rightarrow 0$ in probability if $p \in[1, \infty)$. If $p=\infty$, we use the $\mathcal{F}_{t}$-bounded convergence in probability, meaning that $\operatorname{ess}^{\sup _{\mathcal{H}}}\left\|\xi^{n}\right\|$ is bounded and $\left\|\left(\xi^{n}-\xi\right) \wedge 1\right\|_{1, \mathcal{H}} \rightarrow 0$ in probability as $n \rightarrow \infty$. 
Denote shortly $\mathcal{L}^{p}=\mathcal{L}^{p}\left(\mathbb{R}^{d}, \mathcal{F}\right), \mathcal{L}_{t, s}^{p}=\mathcal{L}_{\mathcal{F}_{t}}^{p}\left(\mathbb{R}^{d}, \mathcal{F}_{s}\right)$ for $t \leq s$, and let $\overline{\mathcal{L}}_{t, s}^{p}=\overline{\mathcal{L}}_{\mathcal{F}_{t}}^{p}\left(\mathbb{R}^{d}, \mathcal{F}_{s}\right)$ be the family of random vectors $\xi_{s}$ that can be decomposed as $\xi_{s}=\xi_{s}^{\prime}+\xi_{s}^{\prime \prime}$, where $\xi_{s}^{\prime} \in \mathcal{L}_{t, s}^{p}$ and $\xi_{s}^{\prime \prime} \in \mathcal{L}^{0}\left(\mathbb{R}_{+}^{d}, \mathcal{F}_{s}\right)$. Following the classical definition of an acceptance set in the theory of risk measures, we introduce the acceptance set $\mathcal{C}_{t, s}$ for $t \leq s$ as the collection of all $\mathcal{F}_{s}$-measurable financial positions regarded acceptable at time $t$.

Definition 2.1 Discrete time $\mathcal{L}^{p}$-dynamic convex acceptance sets are a family $\left\{\mathcal{C}_{t, s}, 0 \leq t \leq s \leq T\right\}$, such that $\mathcal{C}_{t, s} \subset \overline{\mathcal{L}}_{t, s}^{p}$ and the following properties hold for all $0 \leq t \leq s \leq T$.

(i) Normalisation: $\mathcal{C}_{t, t}=\mathcal{L}^{0}\left(\mathbb{R}_{+}^{d}, \mathcal{F}_{t}\right), \mathcal{C}_{t, s} \supset \mathcal{L}^{0}\left(\mathbb{R}_{+}^{d}, \mathcal{F}_{s}\right)$, and $\mathcal{C}_{t, s} \cap \mathcal{L}^{0}\left(\mathbb{R}_{-}^{d}, \mathcal{F}_{t}\right)=\{0\}$.

(ii) Integrability:

$$
\mathcal{C}_{t, s}=\left(\mathcal{C}_{t, s} \cap \mathcal{L}_{t, s}^{p}\right)+\mathcal{L}^{0}\left(\mathbb{R}_{+}^{d}, \mathcal{F}_{s}\right) .
$$

(iii) Closedness: $\mathcal{C}_{t, s} \cap \mathcal{L}_{t, T}^{p}$ is closed in $\mathcal{L}_{t, T}^{p}$.

(iv) Conditional convexity: for all $\alpha_{t} \in \mathcal{L}^{0}\left([0,1], \mathcal{F}_{t}\right)$, and $\eta_{s}^{\prime}, \eta_{s}^{\prime \prime} \in \mathcal{C}_{t, s}$,

$$
\alpha_{t} \eta_{s}^{\prime}+\left(1-\alpha_{t}\right) \eta_{s}^{\prime \prime} \in \mathcal{C}_{t, s}
$$

(v) Weak time consistency: $\mathcal{C}_{t, s} \cap \mathcal{L}^{0}\left(\mathbb{R}^{d}, \mathcal{F}_{u}\right)=\mathcal{C}_{t, u}$ for all $0 \leq t \leq u \leq s \leq T$.

(vi) Compensation: if $\xi_{s} \in \mathcal{L}_{t, s}^{p}$, then $\left(\xi_{s}+\mathcal{C}_{t, s}\right) \cap \mathcal{L}^{0}\left(\mathbb{R}^{d}, \mathcal{F}_{t}\right) \neq \emptyset$.

The integrability property implies that $\mathcal{C}_{t, s}$ is an upper set, that is, $\eta_{s} \in$ $\mathcal{C}_{t, s}$ and $\eta_{s} \leq \eta_{s}^{\prime}$ a.s. (all inequalities are understood coordinatewisely) yield $\eta_{s}^{\prime} \in \mathcal{C}_{t, s}$. The compensation property implies that, for all $\xi_{s} \in \mathcal{L}_{t, s}^{p}$, there exists $\gamma_{t} \in \mathcal{L}^{0}\left(\mathbb{R}^{d}, \mathcal{F}_{t}\right)$ such that $\gamma_{t}+\xi_{s} \in \mathcal{C}_{t, s}$, i.e. it is possible to make the financial position $\xi_{s}$ acceptable by adding the position $\gamma_{t}$. In the following, we need only the acceptance sets $\mathcal{C}_{t-1, t}$ for $t=1, \ldots, T$.

Example 2 (Static univariate convex risk measures) Consider the one-period setting in one dimension with $t=0,1$. If $r$ is a convex $\mathcal{L}^{p}$-risk measure with $p \in[1, \infty)$, then its acceptance set $\mathcal{C}_{0,1} \cap \mathcal{L}^{p}\left(\mathbb{R}, \mathcal{F}_{1}\right)$ is the family of $\eta_{1} \in$ $\mathcal{L}^{p}\left(\mathbb{R}, \mathcal{F}_{1}\right)$ such that $r\left(\eta_{1}\right) \leq 0$. The lower semicontinuity of $r$ is equivalent to the closedness of the acceptance set. The conditional convexity property of the acceptance set is equivalent to the convexity property of the risk measure. The compensation property corresponds to the finiteness of $r$.

The following result refers to the infinite decomposability property (see Definition (9.2), also known as the countable concatenation property [15] or $\sigma$-stability [16].

Lemma 2.1 The families $\mathcal{C}_{t, s}$ are infinitely $\mathcal{F}_{t}$-decomposable for all $0 \leq t \leq$ $s \leq T$.

Proof If $\eta_{s}^{i} \in \mathcal{C}_{t, s} \cap \mathcal{L}_{t, s}^{p}$ and $B_{t}^{i} \in \mathcal{F}_{t}, i \geq 1$, then

$$
\bar{\eta}_{s}^{n}=\sum_{i=1}^{n} \mathbf{1}_{B_{t}^{i}} \eta_{s}^{i}+\eta_{s}^{1} \mathbf{1}_{\Omega \backslash \cup_{i=1}^{n} B_{t}^{i}} \in \mathcal{C}_{t, s}
$$


by the conditional convexity property, so that $\mathcal{C}_{t, s} \cap \mathcal{L}_{t, s}^{p}$ is $\mathcal{F}_{t}$-decomposable. Since $\bar{\eta}_{s}^{n} \rightarrow \bar{\eta}_{s}=\sum_{i=1}^{\infty} \mathbf{1}_{B_{t}^{i}} \eta_{s}^{i}$ in the $\|\cdot\|_{p, \mathcal{F}_{t}}$-norm if $p \in[1, \infty)$ and $\mathcal{F}_{t^{-}}$ boundedly in probability if $p=\infty$, we have $\bar{\eta}_{s} \in \mathcal{C}_{t, s} \cap \mathcal{L}_{t, s}^{p}$. By the integrability property, $\mathcal{C}_{t, s}$ is also infinitely decomposable.

Definition 2.2 The family of dynamic convex acceptance sets is called

(i) coherent if $\alpha_{t} \eta_{s} \in \mathcal{C}_{t, s}$ for all $t \leq s, \alpha_{t} \in \mathcal{L}^{0}\left([0, \infty), \mathcal{F}_{t}\right)$, and $\eta_{s} \in \mathcal{C}_{t, s}$;

(ii) continuous from below at zero if, for all $t \leq s$, and any sequence $\xi_{s}^{n} \in$ $\mathcal{L}_{\mathcal{F}_{t}}^{p}\left(\mathbb{R}_{-}^{d}, \mathcal{F}_{s}\right), n \geq 1$, with $\left\|\xi_{s}^{n}\right\|_{p, \mathcal{F}_{t}} \rightarrow 0$ in probability as $n \rightarrow \infty$, there exists a sequence $\gamma_{t}^{n} \in \mathcal{L}^{0}\left(\mathbb{R}_{+}^{d}, \mathcal{F}_{t}\right), n \geq 1$ and $k \in \mathbb{R}_{+}$, such that $\gamma_{t}^{n}+\xi_{s}^{n} \in$ $\mathcal{C}_{t, s}$ and $\left\|\gamma_{t}^{n}\right\| \leq k\left\|\xi_{s}^{n}\right\|_{p, \mathcal{F}_{t}}$ a.s. for all $n$.

If $p=\infty$ then the continuity from below always holds and is easily verified by choosing $\gamma_{t}^{n}$ with all identical components being $\left\|\xi_{s}^{n}\right\|_{\infty, \mathcal{F}_{t}}$.

Example 3 The acceptance sets can be defined using a univariate convex dynamic $\mathcal{L}^{p}$-risk measure $\left(r_{t}\right)_{t=0, \ldots, T}$, so that $\mathcal{C}_{t, s} \cap \mathcal{L}_{t, s}^{p}$ is the $d$ th Cartesian power of the acceptance set for $r_{t}$. Equivalently, $\xi_{s} \in \mathcal{C}_{t, s} \cap \mathcal{L}_{t, s}^{p}$ if and only if all components of $\xi_{s}$ are acceptable under $r_{t}$. The continuity from below property (with $p \in[1, \infty)$ ) holds if $r_{t}$ is lower semicontinuous in the $\|\cdot\|_{p, \mathcal{F}_{t}}$-norm and continuous from below, which is the case if $r_{t}$ is convex and a.s. finite, see [33, Th. 4.1.4].

Example 4 (Dual construction of conditional acceptance sets) Let $p=\infty$, and consider families $\mathcal{Z}_{t, s} \subset \mathcal{L}_{\mathcal{F}_{t}}^{1}\left(\mathbb{R}_{+}^{d}, \mathcal{F}_{s}\right)$ with $0 \leq t \leq s \leq T$ such that $\mathcal{Z}_{t, u} \subset \mathcal{Z}_{t, s}$ for all $t \leq u \leq s$, and $\mathrm{E}^{g}\left(\zeta_{s} \mid \mathcal{F}_{t}\right)=(1, \ldots, 1)$ for all $\zeta_{s} \in \mathcal{Z}_{t, s}$. Note that we do not assume that $\mathcal{Z}_{t, s}$ is weakly compact. Define

$$
\mathcal{C}_{t, s}=\mathcal{L}^{0}\left(\mathbb{R}_{+}^{d}, \mathcal{F}_{s}\right)+\bigcap_{\zeta_{s} \in \mathcal{Z}_{t, s}}\left\{\eta_{s} \in \mathcal{L}_{t, s}^{\infty}: \mathrm{E}^{g}\left(\left\langle\zeta_{s}, \eta_{s}\right\rangle \mid \mathcal{F}_{t}\right) \geq 0\right\}
$$

where $\left\langle\zeta_{s}, \eta_{s}\right\rangle$ is the scalar product. It is easily seen that conditions (i), (ii), (iv) and (v) of Definition 2.1 hold and these acceptance sets are coherent.

If $\xi_{s}^{n} \rightarrow \xi_{s}$ in probability for $\xi_{s}^{n} \in \mathcal{C}_{t, s}$, and all $\xi_{s}^{n}$ are bounded in the norm by $\gamma_{t} \in \mathcal{L}^{0}\left(\mathbb{R}_{+}, \mathcal{F}_{t}\right)$, then $\mathrm{E}^{g}\left(\left\langle\zeta_{s}, \xi_{s}\right\rangle \mid \mathcal{F}_{t}\right) \geq 0$ by the dominated convergence theorem for generalised conditional expectations. Thus, condition (iii) also hold.

If $\xi_{s} \in \mathcal{L}_{t, s}^{\infty}$, then the components of $\xi_{s}$ are bounded in the absolute value by $\eta_{t} \in \mathcal{L}^{0}\left(\mathbb{R}_{+}^{d}, \mathcal{F}_{t}\right)$. Then, $\eta_{t}-\xi_{s}$ is non-negative and so belongs to $\mathcal{C}_{t, s}$, and $\xi_{s}+\left(\eta_{t}-\xi_{s}\right) \in \mathcal{L}^{0}\left(\mathbb{R}^{d}, \mathcal{F}_{t}\right)$. Thus, (vi) also holds.

\subsection{Dynamic selection risk measures}

Let $\Xi_{T}$ be an upper subset of $\mathcal{L}^{0}\left(\mathbb{R}^{d}, \mathcal{F}_{T}\right)$, that is, with each $\xi \in \Xi_{T}$, the family $\Xi_{T}$ also contains all $\xi^{\prime} \in \mathcal{L}^{0}\left(\xi+\mathbb{R}_{+}^{d}, \mathcal{F}_{T}\right)$. The most important example of such 
family is the family of selections $\mathcal{L}^{0}\left(X_{T}, \mathcal{F}_{T}\right)$ for an $\mathcal{F}_{T}$-measurable 1 upper random set $X_{T}$ in $\mathbb{R}^{d}$, that is, $X_{T}+\mathbb{R}_{+}^{d} \subset X_{T}$ a.s. If $X_{T}$ is also closed, then its centrally symmetric version $\left(-X_{T}\right)$ is a set-valued portfolio in the terminology of 29 .

Definition 2.3 Let $\Xi_{T} \subset \mathcal{L}^{0}\left(\mathbb{R}^{d}, \mathcal{F}_{T}\right)$ be an upper set. For $t \leq s \leq T$,

$$
\mathrm{R}_{t, s}^{0}\left(\Xi_{T}\right)=\left(\Xi_{T}+\mathcal{C}_{t, s}\right) \cap \mathcal{L}^{0}\left(\mathbb{R}^{d}, \mathcal{F}_{t}\right)
$$

denotes the set of all $\gamma_{t} \in \mathcal{L}^{0}\left(\mathbb{R}^{d}, \mathcal{F}_{t}\right)$, such that $\gamma_{t}-\xi \in \mathcal{C}_{t, s}$ for some $\xi \in \Xi_{T}$. Let $\mathrm{R}_{t, s}\left(\Xi_{T}\right)$ denote the closure in probability of $\mathrm{R}_{t, s}^{0}\left(\Xi_{T}\right)$. If $\Xi_{T}=\mathcal{L}^{0}\left(X_{T}, \mathcal{F}_{T}\right)$ is the family of selections of an upper random set $X_{T}$, we write $\mathrm{R}_{t, s}^{0}\left(X_{T}\right)$ and $\mathrm{R}_{t, s}\left(X_{T}\right)$ instead of $\mathrm{R}_{t, s}^{0}\left(\Xi_{T}\right)$ and $\mathrm{R}_{t, s}\left(\Xi_{T}\right)$. In view of this, $\mathrm{R}_{t, s}\left(X_{T}\right)$ (and also $\left.\mathrm{R}_{t, s}\left(\Xi_{T}\right)\right)$ is called the dynamic selection risk measure.

Note that $\mathrm{R}_{T, T}^{0}\left(\Xi_{T}\right)=\Xi_{T}, \mathrm{R}_{t, s}^{0}\left(\Xi_{T}\right)=\mathrm{R}_{t, s}^{0}\left(\Xi_{T} \cap \mathcal{L}^{0}\left(\mathbb{R}^{d}, \mathcal{F}_{s}\right)\right)$, and $\mathrm{R}_{t, u}^{0}\left(\Xi_{T}\right) \subset$ $\mathrm{R}_{t, s}^{0}\left(\Xi_{T}\right)$ for all $0 \leq t \leq u \leq s \leq T$. If only portfolios from a random set $M_{t}$ are allowed for compensation at time $t$, as it is the case in [14, it is easy to modify 2.3 by intersecting $\left(\Xi_{T}+\mathcal{C}_{t, s}\right)$ with $\mathcal{L}^{0}\left(M_{t}, \mathcal{F}_{t}\right)$.

The empty selection risk measure corresponds to completely unacceptable positions. The compensation property of acceptance sets guarantees that $\mathrm{R}_{t, s}^{0}\left(\Xi_{T}\right)$ is not empty if $\Xi_{T} \cap \mathcal{L}_{t, s}^{p} \neq \emptyset$. The family $\Xi_{T}$ is said to be acceptable for the time horizon $s$ if $0 \in \mathrm{R}_{t, s}^{0}\left(\Xi_{T}\right)$, equivalently, $-\Xi_{T}$ contains an element from $\mathcal{C}_{t, s}$. The dynamic selection risk measure is conditionally convex, that is,

$$
\mathrm{R}_{t, s}^{0}\left(\alpha_{t} \Xi_{T}^{\prime}+\left(1-\alpha_{t}\right) \Xi_{T}^{\prime \prime}\right) \supset \alpha_{t} \mathrm{R}_{t, s}^{0}\left(\Xi_{T}^{\prime}\right)+\left(1-\alpha_{t}\right) \mathrm{R}_{t, s}^{0}\left(\Xi_{T}^{\prime \prime}\right)
$$

for all $\alpha_{t} \in \mathcal{L}^{0}\left([0,1], \mathcal{F}_{t}\right)$, and the same holds for the closures. The next result follows from Lemma 2.1 .

Lemma 2.2 If $\Xi_{T}$ is infinitely $\mathcal{F}_{t}$-decomposable, then $\mathrm{R}_{t, s}^{0}\left(\Xi_{T}\right)$ and $\mathrm{R}_{t, s}\left(\Xi_{T}\right)$ are also infinitely $\mathcal{F}_{t}$-decomposable.

Lemma 2.3 Let $X_{T}$ be an $\mathcal{F}_{T}$-measurable random upper closed set.

(i) $\mathrm{R}_{t, s}\left(X_{T}\right)$ is the family of selections of an $\mathcal{F}_{t}$-measurable random upper closed set in $\mathbb{R}^{d}$, which is denoted by $\mathrm{R}_{t, s}\left(X_{T}\right)$.

(ii) If $X_{T}$ is a.s. convex, then $\mathrm{R}_{t, s}^{0}\left(X_{T}\right)$ is a.s. convex. If $X_{T}$ is a cone and the acceptance sets are coherent, then $\mathrm{R}_{t, s}^{0}\left(X_{T}\right)$ is a cone.

Proof (i) By Lemma 2.2. $\mathrm{R}_{t, s}\left(X_{T}\right)$ is an $\mathcal{F}_{t}$-decomposable family, and so Theorem 9.1 applies.

(ii) If $\gamma_{t}^{1}, \gamma_{t}^{2} \in \mathrm{R}_{t, s}^{0}\left(X_{T}\right)$, then $\gamma_{t}^{i}-\xi_{s}^{i} \in \mathcal{C}_{t, s}, i=1,2$, for $\xi_{s}^{1}, \xi_{s}^{2} \in \mathcal{L}^{0}\left(X_{T}, \mathcal{F}_{s}\right)$. For any $t \in(0,1)$, the conditional convexity property yields that $t \gamma_{t}^{1}+(1-$ t) $\gamma_{t}^{2}-\xi_{s} \in \mathcal{C}_{t, s}$ with $\xi_{s}=t \xi_{s}^{1}+(1-t) \xi_{s}^{2} \in \mathcal{L}^{0}\left(X_{T}, \mathcal{F}_{s}\right)$. The conical property is trivial.

Example 5 If $X_{s}=\xi_{s}+\mathbb{R}_{+}^{d}$ for $\xi_{s} \in \mathcal{L}_{t, s}^{p}$, then $\mathrm{R}_{t, s}^{0}\left(X_{s}\right)=\mathrm{R}_{t, s}\left(X_{s}\right)=\mathrm{r}_{t}\left(-\xi_{s}\right)+$ $\mathbb{R}_{+}^{d}$ for a vector-valued dynamic risk measure $\mathrm{r}_{t}$ on $\mathcal{L}_{t, s}^{p}$, see 33 .

\footnotetext{
1 The (graph) measurability of a random set is defined in the appendix.
} 


\section{Hedging to acceptability}

3.1 Acceptable portfolio process

Let $\left(K_{t}\right)_{t=0, \ldots, T}$ be a sequence of random closed convex sets, such that, for all $t$, we have $K_{t} \cap \mathbb{R}_{-}^{d}=\{0\}, K_{t}$ is an upper set, and $K_{t}$ is $\mathcal{F}_{t}$-measurable. The set $K_{t}$ is understood as the family of all solvent positions at time $t$ expressed in physical units and is called a solvency set, see 24. If the solvency sets are cones, this model is well studied under the name of Kabanov's model; it describes the market subject to proportional transaction costs, see 24,32. If the solvency sets are cones and the acceptance sets are coherent, we talk about the coherent conical setting.

Let $K_{t}^{0}$ be the largest $\mathcal{F}_{t}$-measurable linear subspace contained in $K_{t}$, that is,

$$
K_{t}^{0}=\bigcap_{c \neq 0} c K_{t}=\bigcap_{c \in \mathbb{Q} \backslash\{0\}} c K_{t},
$$

which is also a random closed set. The solvency sets are said to be proper if $K_{t}^{0}=\{0\}$ and strictly proper if $\tilde{K}_{t}=K_{t} \cap\left(-K_{t}\right)=\{0\}$ for all $t=0, \ldots, T$. If $K_{t}$ is a cone, then $\tilde{K}_{t}=K_{t}^{0}$, while in general $K_{t}^{0} \subset \tilde{K}_{t}$. Since $\tilde{K}_{t}$ is convex and origin symmetric, $K_{t}$ is proper if and only if $K_{t}$ is bounded.

Definition 3.1 A sequence $V_{t} \in \mathcal{L}^{0}\left(\mathbb{R}^{d}, \mathcal{F}_{t}\right), t=0, \ldots, T$, is called an acceptable portfolio process if

$$
V_{t-1}-V_{t} \in \mathcal{L}^{0}\left(K_{t}, \mathcal{F}_{t}\right)+\mathcal{C}_{t-1, t}, \quad t=1, \ldots, T .
$$

By the definition of the selection risk measure, (3.1) is equivalent to

$$
V_{t-1} \in \mathrm{R}_{t-1, t}^{0}\left(V_{t}+K_{t}\right), \quad t=1, \ldots, T .
$$

Thus, paying transaction costs, it is possible to transform $V_{t-1}-V_{t}$ into an acceptable position for the horizon $t$. Equivalently, $V_{t-1}$ does suffices to purchase $V_{t}+k_{t}+\eta_{t}$ for some $k_{t} \in K_{t}$ and $\eta_{t} \in \mathcal{C}_{t-1, t}$. The initial endowment at time $t$ is any $V_{t-} \in \mathcal{L}^{0}\left(V_{t}+K_{t}, \mathcal{F}_{t}\right)$, so that it is possible to convert $V_{t-}$ into $V_{t}$ paying the transaction costs.

\subsection{Attainable positions and superhedging}

The family of attainable positions at time $s>t$ is the family of random vectors that may be obtained as $V_{s}$ for acceptable portfolio processes starting from zero investment at time $t$. By (3.1), the family of attainable positions is given by

$$
\mathrm{A}_{t, s}=\sum_{u=t}^{s} \mathcal{L}^{0}\left(-K_{u}, \mathcal{F}_{u}\right)-\sum_{u=t}^{s-1} \mathcal{C}_{u, u+1} .
$$

Let $\xi \in \mathcal{L}^{0}\left(\mathbb{R}^{d}, \mathcal{F}_{T}\right)$ be a terminal claim (or payoff). The hedging to acceptability aims to come up with an acceptable portfolio process $\left(V_{t}\right)_{t=0, \ldots, T}$ 
that guarantees paying $\xi$ in the sense that the terminal wealth $V_{T}$ belongs to $\Xi_{T}^{\xi}=\mathcal{L}^{0}\left(X_{T}^{\xi}, \mathcal{F}_{T}\right)$, being the family of selections of the random closed set $X_{T}^{\xi}=\xi+K_{T}$. Define recursively

$$
\Xi_{t}^{\xi}=\mathcal{L}^{0}\left(K_{t}, \mathcal{F}_{t}\right)+\mathrm{R}_{t, t+1}^{0}\left(\Xi_{t+1}^{\xi}\right), \quad t=T-1, \ldots, 0,
$$

which is the set of time $t$ superhedging prices for the claim $\xi$. The family $\Xi_{t}^{\xi}$ consists of the time $t$ superhedging prices for $\xi$ and so may serve as a dynamic convex risk measure of $\xi$ with values being subsets of $\mathcal{L}^{0}\left(\mathbb{R}^{d}, \mathcal{F}_{t}\right)$. If $\xi=\xi^{\prime}-\xi^{\prime \prime}$ for $\xi^{\prime} \in \mathcal{L}^{p}\left(\mathbb{R}^{d}, \mathcal{F}_{T}\right)$ and $\xi^{\prime \prime} \in \mathcal{L}^{0}\left(\mathbb{R}_{+}^{d}, \mathcal{F}_{T}\right)$, the compensation property of acceptance sets ensures that $\Xi_{t}^{\xi}$ is not empty for all $t$.

In order to handle the asymptotic version of the risk measure, let $\hat{\Xi}_{T}^{\xi}=\Xi_{T}^{\xi}$, and further

$$
\hat{\Xi}_{t}^{\xi}=\mathcal{L}^{0}\left(K_{t}, \mathcal{F}_{t}\right)+\mathrm{R}_{t, t+1}\left(\Xi_{t+1}^{\xi}\right), \quad t=T-1, \ldots, 0 .
$$

Note that $\Xi_{t}^{\xi} \subset \hat{\Xi}_{t}^{\xi} \subset \operatorname{cl}_{0}\left(\Xi_{t}^{\xi}\right)$, whence $\operatorname{cl}_{0}\left(\hat{\Xi}_{t}^{\xi}\right)=\operatorname{cl}_{0}\left(\Xi_{t}^{\xi}\right)$ for all $t$. The families $\Xi_{t}^{0}$ and $\hat{\Xi}_{T}^{0}$ arise by letting $\xi=0$ a.s.

Lemma 3.1 (i) The families $\mathrm{R}_{t, s}^{0}\left(\Xi_{s}^{\xi}\right), \mathrm{R}_{t, s}\left(\Xi_{s}^{\xi}\right)$, and $\hat{\Xi}_{t}^{\xi}$ are convex and infinitely $\mathcal{F}_{t}$-decomposable for all $0 \leq t \leq s \leq T$.

(ii) For each $t \leq T$, there exists a (possibly non-closed) random set $X_{t}^{\xi}$ such that $\hat{\Xi}_{t}^{\xi}=\mathcal{L}^{0}\left(X_{t}^{\xi}, \mathcal{F}_{t}\right)$.

(iii) For any $t \leq T$, the family of all initial endowments $V_{t-}$ at time $t$, allowing to start an acceptable portfolio process $\left(V_{s}\right)_{t \leq s \leq T}$ such that $V_{T} \in$ $\mathcal{L}^{0}\left(\xi+K_{T}, \mathcal{F}_{T}\right)$ a.s., coincides with $\Xi_{t}^{\xi}$ and

$$
\Xi_{t}^{\xi}=\left(-\mathrm{A}_{t, T}+\xi\right) \cap \mathcal{L}^{0}\left(\mathbb{R}^{d}, \mathcal{F}_{t}\right) .
$$

(iv) If $K_{T}$ is a cone, then $\Xi_{t}^{\xi} \subset \Xi_{t}^{0}$ for any $\xi \in \mathcal{L}^{0}\left(K_{T}, \mathcal{F}_{T}\right)$.

Proof (i) follows from Lemma 2.2 .

(ii) The existence of $X_{t}^{\xi}$ is trivial for $t=T$. Suppose that it holds at time $t$. The result for $t-1$ follows from the induction assumption and (3.4) by Lemma 9.1

(iii) follows from the fact that $\left(\gamma_{T}+\mathrm{A}_{t, T}\right) \cap\left(\xi+K_{T}\right) \neq \emptyset$ if and only if $\gamma_{T} \in\left(-\mathrm{A}_{t, T}+\xi\right)$.

(iv) follows from (iii), since $\xi+K_{T} \subset K_{T}$ a.s.

Example 6 If $K_{t}=\mathbb{R}_{+}^{d}$ a.s. for all $t$ (which is always the case if $d=1$ ), then an acceptable portfolio process satisfies $V_{t-1}-V_{t} \in \mathcal{C}_{t-1, t}$ for all $t=1, \ldots, T$. Then,

$$
\Xi_{t}^{\xi}=\left(\xi+\sum_{s=t}^{T-1} \mathcal{C}_{s, s+1}\right) \cap \mathcal{L}^{0}\left(\mathbb{R}^{d}, \mathcal{F}_{t}\right) .
$$

Since $\mathcal{C}_{t-1, t} \cap \mathcal{L}^{0}\left(\mathbb{R}^{d}, \mathcal{F}_{t-1}\right)=\mathcal{L}^{0}\left(\mathbb{R}_{+}^{d}, \mathcal{F}_{t-1}\right)$ for all $t \geq 1$ by the weak time consistency and normalisation properties, the induction argument yields that 
$\Xi_{t}^{0}=\mathcal{L}^{0}\left(\mathbb{R}_{+}^{d}, \mathcal{F}_{t}\right)$. If $\xi$ does not a.s. vanish, the set $\mathrm{R}_{t, s}^{0}\left(\Xi_{T}^{\xi}\right)$ becomes nontrivial. Its static variant is called a regulator risk measure in 20]; it only takes into account the acceptability requirement and disregards any trading opportunities between the components. In the terminology of [20], $\mathrm{R}_{0,1}\left(\xi+K_{1}\right)$ (in the static setting with a conical $K_{1}$ ) is called the market extension of the regulator risk measure.

\section{Risk arbitrage}

Recall that $\Xi_{t}^{0}$ is the set of time $t$ super-hedging prices for the zero claim. By (3.5),

$$
\Xi_{t}^{0}=\left(-\mathrm{A}_{t, T}\right) \cap \mathcal{L}^{0}\left(\mathbb{R}^{d}, \mathcal{F}_{t}\right)
$$

For multivariate financial models, e.g. models with proportional transaction costs, several no arbitrage conditions have been considered. In Kabanov's model, there is the NA condition, its robust version $\mathrm{NA}^{r}$, but also the NA2 condition derived using an alternative approach, see 31 and 24. All these conditions are formulated in terms of the set of all terminal claims $\mathrm{A}_{t, T}$ attainable from the zero initial endowment. Here, we consider weaker no arbitrage conditions imposed on the super-hedging prices for the zero claim.

Definition 4.1 The multiperiod model satisfies

(SNR) (strict no risk arbitrage) if $\hat{\Xi}_{t}^{0} \cap \mathcal{L}^{0}\left(-K_{t}, \mathcal{F}_{t}\right) \subset \mathcal{L}^{0}\left(K_{t}^{0}, \mathcal{F}_{t}\right)$ for all $t=0, \ldots, T$

(NRA) (no risk arbitrage) if $\Xi_{t}^{0} \cap \mathcal{L}^{0}\left(\mathbb{R}_{-}^{d}, \mathcal{F}_{t}\right)=\{0\}$ for all $t=0, \ldots, T$;

(NARA) (no asymptotic risk arbitrage) if $\left(\mathrm{cl}_{0} \Xi_{t}^{0}\right) \cap \mathcal{L}^{0}\left(\mathbb{R}_{-}^{d}, \mathcal{F}_{t}\right)=\{0\}$ for all $t=0, \ldots, T$

(NRA2) (no risk arbitrage opportunity of the second kind) if, for any $t=$ $0, \ldots, T$ and $\eta_{t} \in \mathcal{L}^{0}\left(\mathbb{R}^{d}, \mathcal{F}_{t}\right)$ such that $\left(\eta_{t}+\mathrm{A}_{t, T}\right) \cap \mathcal{L}^{0}\left(K_{T}, \mathcal{F}_{T}\right) \neq \emptyset$, we have $\eta_{t} \in \mathcal{L}^{0}\left(K_{t}, \mathcal{F}_{t}\right)+\mathcal{C}_{t-1, t}$;

(SNAR) (strong no risk arbitrage) if $\sum_{t=0}^{T}\left(k_{t}+\eta_{t}\right)=0$ for $k_{t} \in \mathcal{L}^{0}\left(K_{t}, \mathcal{F}_{t}\right)$ and $\eta_{t} \in \mathcal{C}_{t-1, t}$ for all $t$ implies that $k_{t} \in \mathcal{L}^{0}\left(K_{t}^{0}, \mathcal{F}_{t}\right)$ and $\eta_{t}=0$ a.s. for all $t$.

Let us comment on the $(\mathrm{SNR})$ condition. If $p_{t} \in \hat{\Xi}_{t}^{0} \cap \mathcal{L}^{0}\left(-K_{t}, \mathcal{F}_{t}\right)$, then, starting from the zero initial endowment at time $t$ expressed as $0=p_{t}-$ $p_{t}$, we obtain the zero claim at time $T$ from the price $p_{t}$ and allowing an immediate possible profit at time $t$, since the liquidation value of $-p_{t} \in K_{t}$ is non-negative. A similar interpretation applies for the (NRA) condition and its asymptotic version (NARA). The (NRA2) condition may be compared to the (NA2) condition of 31, while (SNAR) is a version of 24, Condition (iii), Section 3.2.2]. Note that (NRA) condition is equivalent to $A_{t, T} \cap \mathcal{L}^{0}\left(\mathbb{R}_{+}^{d}, \mathcal{F}_{t}\right)=$ $\{0\}$, while the usual (NA) condition $A_{t, T} \cap \mathcal{L}^{0}\left(\mathbb{R}_{+}^{d}, \mathcal{F}_{T}\right)=\{0\}$ is stronger, see 24, Section 3.2.1].

Example 1 shows that it may be possible to release infinite capital from zero position without compromising the acceptability criterion, in particular, 
it violates the (SNR) condition. By Lemma 3.1. (SNR) can be written as $X_{t}^{0} \cap\left(-K_{t}\right) \subset K_{t}^{0}$ a.s., and (NARA) as $\left(\operatorname{cl} X_{t}^{0}\right) \cap \mathbb{R}_{-}^{d}=\{0\}$ a.s. It is obvious that (NARA) is stronger than (NRA). By (4.1), (NRA) condition is equivalent to $\mathrm{A}_{t, T} \cap \mathcal{L}^{0}\left(\mathbb{R}_{+}^{d}, \mathcal{F}_{t}\right)=\{0\}$ for all $t$. If $K_{t}=\mathbb{R}_{+}^{d}$ a.s. for all $t$, then $\Xi_{t}^{0}=\mathcal{L}^{0}\left(\mathbb{R}_{+}^{d}, \mathcal{F}_{t}\right)$ and all no arbitrage conditions are satisfied, see Example 6 .

Lemma 4.1 (SNR) implies that

$$
\mathrm{R}_{t, t+1}\left(\Xi_{t+1}^{0}\right) \cap \mathcal{L}^{0}\left(-K_{t}, \mathcal{F}_{t}\right) \subset \mathcal{L}^{0}\left(K_{t}^{0}, \mathcal{F}_{t}\right), \quad t=0, \ldots, T-1 .
$$

The reverse implication holds if the solvency sets are strictly proper.

Proof Denote $M=\mathrm{R}_{t, t+1}\left(\Xi_{t+1}^{0}\right), A=\mathcal{L}^{0}\left(K_{t}, \mathcal{F}_{t}\right)$, and $B=\mathcal{L}^{0}\left(K_{t}^{0}, \mathcal{F}_{t}\right)$. It is easily seen that $M \cap(-A) \subset B$ if $(M+A) \cap(-A) \subset B$ and only if in case $A \cap(-A)=\{0\}$. For the reverse implication, if $x \in(M+A) \cap(-A)$, then $x=$ $m+a_{1}=-a_{2}$, where $m \in M$ and $a_{1}, a_{2} \in A$. Therefore, $m / 2 \in M \cap(-A) \subset B$. Then $x / 2 \in A \cap(-A)$, so that $x \in B=A \cap(-A)=\{0\}$ if $K$ is strictly proper.

Lemma 4.2 Assume that the acceptance sets are strictly proper, that is, $\mathcal{C}_{t, s} \cap$ $\left(-\mathcal{C}_{t, s}\right)$ consists of all random vectors that equal 0 almost surely.

(i) If $K_{t}^{0}=\tilde{K}_{t}$ for all $t$, (SNAR) implies

$$
\begin{array}{ll}
\mathrm{A}_{0, t} \cap\left(\mathcal{L}^{0}\left(K_{t}, \mathcal{F}_{t}\right)+\mathcal{C}_{t-1, t}\right) \subset \mathcal{L}^{0}\left(K_{t}^{0}, \mathcal{F}_{t}\right), & t=0, \ldots, T, \\
\mathrm{~A}_{t, T} \cap\left(\mathcal{L}^{0}\left(K_{t}, \mathcal{F}_{t}\right)+\mathcal{C}_{t-1, t}\right) \subset \mathcal{L}^{0}\left(K_{t}^{0}, \mathcal{F}_{t}\right), & t=0, \ldots, T .
\end{array}
$$

(ii) If the solvency sets are strictly proper and

$$
\mathcal{L}^{0}\left(-K_{t}, \mathcal{F}_{t}\right) \cap \mathcal{C}_{t-1, t}=\{0\}, \quad t=0, \ldots, T,
$$

then either of the conditions (4.2), (4.3) implies (SNAR).

Proof (i) Motivated by [24, Lemma 3.2.7], assume that

$$
-k_{0}-\cdots-k_{t}-\eta_{0}-\cdots-\eta_{t}=g_{t}+\zeta_{t} \in \mathrm{A}_{0, t} \cap\left(\mathcal{L}^{0}\left(K_{t}, \mathcal{F}_{t}\right)+\mathcal{C}_{t-1, t}\right)
$$

with $k_{s} \in \mathcal{L}^{0}\left(K_{s}, \mathcal{F}_{s}\right), \eta_{s} \in \mathcal{C}_{s-1, s}, s=0, \ldots, t, g_{t} \in \mathcal{L}^{0}\left(K_{t}, \mathcal{F}_{t}\right)$, and $\zeta_{t} \in$ $\mathcal{C}_{t-1, t}$. Since $\left(\eta_{t}+\zeta_{t}\right) / 2 \in \mathcal{C}_{t-1, t}$ by convexity and

$$
-k_{0} / 2-\cdots-k_{t-1} / 2-\left(k_{t}+g_{t}\right) / 2-\eta_{0} / 2-\cdots-\left(\eta_{t}+\zeta_{t}\right) / 2=0,
$$

we have $\left(k_{t}+g_{t}\right) / 2 \in K_{t}^{0}$ and $\left(\eta_{t}+\zeta_{t}\right) / 2=0$ by (SNAR). The strict properness of the acceptance sets yields that $\eta_{t}=\zeta_{t}=0$. Furthermore, $\frac{1}{2} g_{t} \in-\frac{1}{2} k_{t}+$ $\frac{1}{2} K_{t}^{0} \subset-K_{t}$, so that $g_{t} \in K_{t}^{0}$, i.e. (4.2) holds.

Property (4.3) is similarly derived from (SNAR).

(ii) In order to show that (4.2) implies (SNAR), proceed by induction as in [24, Lemma 3.2.13]. Let $-k_{0}-\cdots-k_{T}-\eta_{0}-\cdots-\eta_{T}=0$. Then

$$
k_{T}+\eta_{T}=\sum_{s=0}^{T-1}\left(-k_{s}-\eta_{s}\right) \in \mathrm{A}_{0, T-1} \subset \mathrm{A}_{0, T} .
$$


By (4.2), $k_{T}+\eta_{T} \in \mathcal{L}^{0}\left(K_{T}^{0}, \mathcal{F}_{T}\right)$. Since $k_{T}+\eta_{T}$ is $\mathcal{F}_{T-1}$-measurable and the solvency sets are strictly proper, $k_{T}+\eta_{T} \in \mathcal{L}^{0}\left(K_{T-1}^{0}, \mathcal{F}_{T-1}\right)$. Therefore, $k_{T}+\eta_{T}$ can be merged with $k_{T-1}$, and then the induction proceeds with $T-1$ instead of $T$.

To show that (4.3) implies (SNAR), proceed by induction starting from time zero. Since

$$
k_{0}+\eta_{0}=\sum_{s=1}^{T}\left(-k_{s}-\eta_{s}\right) \in \mathrm{A}_{1, T} \subset \mathrm{A}_{0, T},
$$

(4.3) yields that $k_{0}+\eta_{0}=0$, and (4.4) implies $k_{0}=\eta_{0}=0$.

Condition (4.4) can be viewed as a consistency between the acceptance sets and solvency sets, namely, that $-K_{t}$ does not contain any acceptable non-trivial selection.

The first part of the following result shows that (NRA) is similar to the weak no arbitrage property $\mathrm{NA}^{\mathrm{w}}$ of Kabanov's model, see [24, Sec. 3.2.1]. Denote by $\operatorname{int} A$ the interior and by $\partial A$ the boundary of $A \subset \mathbb{R}^{d}$.

Proposition 4.1 Suppose that $\mathbb{R}_{+}^{d} \backslash\{0\} \subset \operatorname{int} K_{t}$ a.s. for all $t$. Then (NRA) is equivalent to each of the following two conditions.

(i) $\mathrm{R}_{t, t+1}^{0}\left(\Xi_{t+1}^{0}\right) \cap \mathcal{L}^{0}\left(-K_{t}, \mathcal{F}_{t}\right) \subset \mathcal{L}^{0}\left(-\partial K_{t}, \mathcal{F}_{t}\right)$ for all $t$.

(ii) $\hat{\Xi}_{t}^{0} \cap \mathcal{L}^{0}\left(\mathbb{R}_{-}^{d}, \mathcal{F}_{t}\right)=\{0\}$ for all $t$.

Proof (i) Consider $x_{t}=\gamma_{t}+k_{t}$ for $\gamma_{t} \in M=\mathrm{R}_{t, t+1}^{0}\left(\Xi_{t+1}^{0}\right)$ and $k_{t} \in \mathcal{L}^{0}\left(K_{t}, \mathcal{F}_{t}\right)$. Assume that $x_{t}$ is non-trivial and $x_{t} \in \mathcal{L}^{0}\left(\mathbb{R}_{-}^{d}, \mathcal{F}_{t}\right)$. Hence, $\gamma_{t} / 2=x_{t} / 2-k_{t} / 2 \in$ $\mathcal{L}^{0}\left(-K_{t}, \mathcal{F}_{t}\right)$ and $\gamma_{t} / 2 \in-\operatorname{int} K_{t}$ on $\left\{x_{t} \neq 0\right\}$, since $\operatorname{int} K_{t}$ contains $\mathbb{R}_{+}^{d} \backslash\{0\}$, contrary to the assumption.

Consider any $x_{t} \in M \cap \mathcal{L}^{0}\left(-K_{t}, \mathcal{F}_{t}\right)$ such that $x_{t}=-k_{t}$ for $k_{t} \in \mathcal{L}^{0}\left(K_{t}, \mathcal{F}_{t}\right)$ such that $\mathrm{P}\left(k_{t} \in \operatorname{int} K_{t}\right)>0$. By a measurable selection argument, $k_{t}+\gamma_{t} \in$ $\mathcal{L}^{0}\left(K_{t}, \mathcal{F}_{t}\right)$ for some $\gamma_{t} \in \mathcal{L}^{0}\left(\mathbb{R}_{-}^{d}, \mathcal{F}_{t}\right) \backslash\{0\}$. Thus,

$$
x_{t}+k_{t}+\gamma_{t}=\gamma_{t} \in\left(M+\mathcal{L}^{0}\left(K_{t}, \mathcal{F}_{t}\right)\right) \cap \mathcal{L}^{0}\left(\mathbb{R}_{-}^{d}, \mathcal{F}_{t}\right)
$$

contrary to (NRA).

(ii) It suffices to show that (NRA) implies (ii). Assume that $k_{t} \in \mathcal{L}^{0}\left(K_{t}, \mathcal{F}_{t}\right)$ and $\gamma_{t} \in \mathrm{R}_{t, t+1}\left(\Xi_{t+1}^{0}\right)$ are such that $k_{t}+\gamma_{t} \in \mathbb{R}_{-}^{d}$ a.s. and $k_{t}+\gamma_{t} \neq 0$ with a positive probability. Since $k_{t} / 2+\mathbb{R}_{+}^{d} \subset\left(\left\{k_{t} / 2\right\} \cup \operatorname{int} K_{t}\right)$ a.s., the set (int $K_{t}+$ $\left.\mathrm{R}_{t, t+1}\left(\Xi_{t+1}^{0}\right)\right)$ with a positive probability has a non-trivial intersection with $\mathbb{R}_{-}^{d}$. By [26, Prop. 2.10] with $X=\operatorname{int} K_{t}$ and $\Xi=\mathrm{R}_{t, t+1}^{0}\left(\Xi_{t+1}^{0}\right)$, the set (int $K_{t}+$ $\left.\mathrm{R}_{t, t+1}^{0}\left(\Xi_{t+1}^{0}\right)\right)$ has a non-trivial intersection with $\mathbb{R}_{-}^{d}$ with a positive probability, contrary to (NRA).

Theorem 4.1 If the solvency sets are proper, then (SNR) implies (NARA) and the closedness of $\hat{\Xi}_{t}^{0}$ in probability for all $t=0, \ldots, T$. 
Proof Denote $M=\mathrm{R}_{t, t+1}\left(\Xi_{t+1}^{0}\right)$. Recall that $\mathrm{cl}_{0} \hat{\Xi}_{t}^{0}=\mathrm{cl}_{0} \Xi_{t}^{0}$. Assume that $k_{t}^{n}+\gamma_{t}^{n} \rightarrow \zeta_{t} \in \mathcal{L}^{0}\left(\mathbb{R}_{-}^{d}, \mathcal{F}_{t}\right)$ a.s. for $k_{t}^{n} \in \mathcal{L}^{0}\left(K_{t}, \mathcal{F}_{t}\right)$ and $\gamma_{t}^{n} \in M$ such that $k_{t}^{n}+\gamma_{t}^{n} \in \hat{\Xi}_{t}^{0}, n \geq 1$. Since $M$ is $\mathcal{L}^{0}$-closed and convex, we may assume by [24, Lemma 2.1.2] that $k_{t}^{n} \rightarrow k_{t} \in \mathcal{L}^{0}\left(K_{t}, \mathcal{F}_{t}\right)$ on the set $A=\left\{\liminf _{n}\left\|k_{t}^{n}\right\|<\infty\right\}$. Hence, $\gamma_{t}^{n} \rightarrow \gamma_{t} \in M$, so that

$$
\gamma_{t}=\zeta_{t}-k_{t} \in M \cap \mathcal{L}^{0}\left(-K_{t}, \mathcal{F}_{t}\right) \subset \mathcal{L}^{0}\left(K_{t}^{0}, \mathcal{F}_{t}\right) .
$$

Thus, $\gamma_{t} \in K_{t}^{0}$ and $\zeta_{t} / 2=\gamma_{t} / 2+k_{t} / 2 \in K_{t}$. Hence, $\zeta_{t} / 2 \in \mathbb{R}_{-}^{d} \cap K_{t}=\{0\}$ and $\zeta_{t}=0$ on $A$.

If $\mathrm{P}(\Omega \backslash A)>0$, assume that $k_{t}^{n}=\gamma_{t}^{n}=\zeta_{t}=0$ on $A$ by $\mathcal{F}_{t}$-decomposability, and use the standard normalisation procedure, i.e. divide $k_{t}^{n}, \gamma_{t}^{n}, \zeta_{t}$ by $(1+$ $\left.\left\|k_{t}^{n}\right\|\right)$. Arguing as previously, we obtain $k_{t} \in \mathcal{L}^{0}\left(K_{t}, \mathcal{F}_{t}\right)$ such that $\left\|k_{t}\right\|=1$ on $\Omega \backslash A$. Since $0 \in M$, we have $\gamma_{t} \in M$ by conditional convexity. Moreover, $k_{t}+\gamma_{t}=0$ since $\zeta_{t} /\left(1+\left\|k_{t}^{n}\right\|\right) \rightarrow 0$. Hence, $\gamma_{t} \neq 0$ belongs to $M \cap \mathcal{L}^{0}\left(-K_{t}, \mathcal{F}_{t}\right)=$ $\{0\}$, which is a contradiction in view of Lemma 4.1

This argument also yields the closedness of $\hat{\Xi}_{t}^{0}=\mathcal{L}^{0}\left(K_{t}, \mathcal{F}_{t}\right)+M$.

Let $\overline{\mathrm{A}}_{t, s}^{p}$ denote the closure of $\mathrm{A}_{t, s}^{p}=\mathrm{A}_{t, s} \cap \mathcal{L}^{p}\left(\mathbb{R}^{d}, \mathcal{F}_{T}\right)$ in $\mathcal{L}^{p}$ for $p<\infty$ and in the $\mathcal{F}_{t}$-bounded convergence in probability for $p=\infty$.

The following theorem states that the (NARA) and (SNR) conditions are weak no arbitrage conditions of the no free lunch type. Recall that the usual NFL condition is $\overline{\mathrm{A}}_{t, T}^{p} \cap \mathcal{L}^{0}\left(\mathbb{R}_{+}^{d}, \mathcal{F}_{T}\right)=\{0\}$.

Theorem 4.2 Assume that the acceptance sets are continuous from below and $p \in[1, \infty]$.

(i) (NARA) is equivalent to

$$
\overline{\mathrm{A}}_{t, T}^{p} \cap \mathcal{L}^{0}\left(\mathbb{R}_{+}^{d}, \mathcal{F}_{t}\right)=\{0\}, \quad t=0, \ldots, T-1 .
$$

(ii) If the solvency sets are proper, then (SNR) is equivalent to

$$
\overline{\mathrm{A}}_{t, T}^{p} \cap \mathcal{L}^{0}\left(K_{t}, \mathcal{F}_{t}\right)=\{0\}, \quad t=0, \ldots, T-1 .
$$

Moreover, properties (4.5) and (4.6) are equivalent to the same ones with $p=1$ and also to those obtained by taking the closure of $\mathrm{A}_{t, T}$ in $\mathcal{L}_{t, T}^{p}=\mathcal{L}_{\mathcal{F}_{t}}^{p}\left(\mathbb{R}^{d}, \mathcal{F}_{T}\right)$.

Proof (i) Assume that (4.5) holds for the closure with respect to the conditional norm on $\mathcal{L}_{t, T}^{p}$. Thus, (4.5) also holds if the closure is taken with respect to the norm on $\mathcal{L}^{p}\left(\mathbb{R}^{d}, \mathcal{F}_{T}\right)$. Therefore, given (4.1), it suffices to show that (NARA) follows from

$$
\operatorname{cl}_{p}\left(\Xi_{t}^{0} \cap \mathcal{L}^{p}\left(\mathbb{R}^{d}, \mathcal{F}_{t}\right)\right) \cap \mathcal{L}^{0}\left(\mathbb{R}_{-}^{d}, \mathcal{F}_{t}\right)=\{0\}, \quad t=0, \ldots, T-1 .
$$

Assume (4.7) and consider $x_{t} \in\left(\mathrm{cl}_{0} \Xi_{t}^{0}\right) \cap \mathcal{L}^{0}\left(\mathbb{R}_{-}^{d}, \mathcal{F}_{t}\right)$. Then $x_{t}^{n} \rightarrow x_{t}$ a.s. for $x_{t}^{n} \in \Xi_{t}^{0}, n \geq 1$. Hence,

$$
x_{t}^{n} \mathbf{1}_{\left\|x_{t}^{n}\right\| \leq m+1} \mathbf{1}_{\left\|x_{t}\right\| \leq m} \rightarrow x_{t} \mathbf{1}_{\left\|x_{t}\right\| \leq m} \quad \text { a.s. as } n \rightarrow \infty
$$


for all $m \geq 1$, where $x_{t}^{n} \mathbf{1}_{\left\|x_{t}^{n}\right\| \leq m+1} \mathbf{1}_{\left\|x_{t}\right\| \leq m} \in \Xi_{t}^{0}$ by decomposability and since $0 \in \Xi_{t}^{0}$. The dominated convergence theorem yields that $x_{t} \mathbf{1}_{\left\|x_{t}\right\| \leq m}$ belongs to $\operatorname{cl}_{p}\left(\Xi_{t}^{0} \cap \mathcal{L}^{p}\left(\mathbb{R}^{d}, \mathcal{F}_{t}\right)\right) \cap \mathcal{L}^{0}\left(\mathbb{R}_{-}^{d}, \mathcal{F}_{t}\right)=\{0\}$, where the closure may be taken with respect to the conditional norm. Letting $m \rightarrow \infty$ yields $x_{t}=0$, i.e. (NARA) holds.

Assume (NARA). Consider a sequence $\left(V_{t, T}^{n}\right)_{n \geq 1}$ from $\mathrm{A}_{t, T}^{p}$ which converges in $\mathcal{L}_{t, T}^{p}$ to $z_{t}^{+} \in \mathcal{L}^{p}\left(\mathbb{R}_{+}^{d}, \mathcal{F}_{t}\right)$. Then, $\tilde{V}_{t, T}^{n}=V_{t, T}^{n} \wedge z_{t}^{+} \rightarrow z_{t}^{+}$in $\mathcal{L}_{u, T}^{p}$, where the minimum is taken coordinatewisely and $u$ is any time moment between $t$ and $T$, and $\tilde{V}_{t, T}^{n} \in \mathrm{A}_{t, T}$, so that we may assume without loss of generality that $V_{t, T}^{n} \leq z_{t}^{+}$. Passing to subsequences, assume that $V_{t, T}^{n} \rightarrow z_{t}^{+}$in $\mathcal{L}_{u, T}^{p}$ and almost surely for each given $u \geq t$.

Define $\xi_{T}^{n}=V_{t, T}^{n}-z_{t}^{+} \leq 0$. Then $\left\|\xi_{T}^{n}\right\|_{p, \mathcal{F}_{T-1}} \rightarrow 0$ in probability. By the continuity from below, there exists a sequence $\gamma_{T-1}^{n} \in \mathcal{L}^{0}\left(\mathbb{R}_{+}^{d}, \mathcal{F}_{T-1}\right), n \geq 1$, such that

$$
\eta_{T}^{n}=\xi_{T}^{n}+\gamma_{T-1}^{n} \in \mathcal{C}_{T-1, T}
$$

and $0 \leq \gamma_{T-1}^{n} \leq x_{T}\left\|\xi_{T}^{n}\right\|_{p, \mathcal{F}_{T-1}}$ for all $n$ and some $x_{T} \in \mathbb{R}_{+}^{d}$. Hence, $\gamma_{T-1}^{n} \rightarrow 0$ in $\mathcal{L}_{T-2, T}^{p}$ if $T-2 \geq t$. Since $-\gamma_{T-1}^{n} \rightarrow 0$ in $\mathcal{L}_{T-2, T}^{p}$, the continuity from below property yields the existence of a sequence $\gamma_{T-2}^{n} \in \mathcal{L}^{0}\left(\mathbb{R}_{+}^{d}, \mathcal{F}_{T-2}\right)$ such that

$$
\eta_{T-1}^{n}=-\gamma_{T-1}^{n}+\gamma_{T-2}^{n} \in \mathcal{C}_{T-2, T-1}
$$

for all $n$, and, for some constant $x_{T-1} \in \mathbb{R}_{+}^{d}$, we have

$$
0 \leq \gamma_{T-2}^{n} \leq x_{T-1}\left\|\gamma_{T-1}^{n}\right\|_{p, \mathcal{F}_{T-1}} \leq x_{T} x_{T-1}\left\|\xi_{T}^{n}\right\|_{p, \mathcal{F}_{T-2}},
$$

so that $\gamma_{T-2}^{n} \rightarrow 0$ in $\mathcal{L}_{T-3, T}^{p}$ if $T-3 \geq t$. Iterate the construction to find $\gamma_{T-3}^{n}, \ldots, \gamma_{t}^{n}$ such that $\gamma_{t}^{n} \rightarrow 0$ a.s. Then $\eta_{u+1}^{n}=-\gamma_{u+1}^{n}+\gamma_{u}^{n} \in \mathcal{C}_{u, u+1}$ if $t \leq u \leq T-2$. Hence,

$$
\xi_{T}^{n}+\gamma_{t}^{n}=\sum_{u=t}^{T-1} \eta_{u+1}^{n} \in \mathcal{C}_{t, t+1}+\cdots+\mathcal{C}_{T-1, T} .
$$

By convexity,

$$
\frac{1}{2}\left(-z_{t}^{+}+\gamma_{t}^{n}\right)=-\frac{1}{2} V_{t, T}^{n}+\frac{1}{2}\left(\xi_{T}^{n}+\gamma_{t}^{n}\right) \in \Xi_{t}^{0} .
$$

Letting $n \rightarrow \infty$ yields that $-\frac{1}{2} z_{t}^{+} \in\left(\mathrm{cl}_{0} \Xi_{t}^{0}\right) \cap \mathcal{L}^{0}\left(-\mathbb{R}_{+}^{d}, \mathcal{F}_{t}\right)$, so that $z_{t}^{+}=0$ by (NARA). Thus, (4.5) holds with respect to the conditional norm and also with respect to the $\mathcal{L}^{p}$-norm.

(ii) Recall that $\hat{\Xi}_{t}^{0}=\operatorname{cl}_{0}\left(\hat{\Xi}_{t}^{0}\right)=\mathrm{cl}_{0}\left(\Xi_{t}^{0}\right)$ by Theorem 4.1 Following the arguments from (i), we obtain that (SNR) is equivalent to

$$
\operatorname{cl}_{p}\left(\Xi_{t}^{0} \cap \mathcal{L}^{p}\left(\mathbb{R}^{d}, \mathcal{F}_{t}\right)\right) \cap \mathcal{L}^{p}\left(-K_{t}, \mathcal{F}_{t}\right)=\{0\}, \quad t=0, \ldots, T-1 .
$$

In view of (4.1), $\operatorname{cl}_{p}\left(\Xi_{t}^{0} \cap \mathcal{L}^{p}\right) \subset-\overline{\mathrm{A}}_{t, T}^{p}$. Therefore, (4.6) implies (4.8) and (SNR) holds. 
Now assume (SNR). Consider a sequence $\left(V_{t, T}^{n}\right)_{n \geq 1}$ from $\mathrm{A}_{t, T}^{p}$ which converges in $\mathcal{L}^{p}$ to $k_{t} \in \mathcal{L}^{0}\left(K_{t}, \mathcal{F}_{t}\right)$. Then follow the proof of (i) with $k_{t}$ instead of $z_{t}^{+}$.

Consider a sequence $\left(V_{t, T}^{n}\right)_{n \geq 1}$ from $\mathrm{A}_{t, T}^{1}$ which converges in $\mathcal{L}^{1}$ to $k_{t} \in$ $\mathcal{L}^{0}\left(K_{t}, \mathcal{F}_{t}\right)$. We may assume that $\left(V_{t, T}^{n}\right)_{n \geq 1}$ converges a.s. Then, for every $M>0,\left(V_{t, T}^{n} \mathbf{1}_{\left\|k_{t}\right\| \leq M}\right)_{n \geq 1}$ is a sequence from $\mathrm{A}_{t, T}^{1}$ which converges in $\mathcal{L}^{1}$ to $k_{t} \mathbf{1}_{\left\|k_{t}\right\| \leq M} \in \mathcal{L}^{p}\left(\bar{K}_{t}, \mathcal{F}_{t}\right)$ so that we may assume without loss of generality that $\left\|k_{t}\right\|$ is bounded by $M$. Passing to a subsequence, assume that $\mathrm{E}\left(\| V_{t, T}^{n}-\right.$ $\left.k_{t} \| \mid \mathcal{F}_{t}\right) \rightarrow 0$ a.s. Therefore, $V_{t, T}^{n} \mathbf{1}_{\mathrm{E}\left(\left\|V_{t, T}^{n}\right\| \mid \mathcal{F}_{t}\right) \leq M+1} \rightarrow k_{t}$ almost surely and in $\mathcal{L}_{t, T}^{1}$. Thus, 4.6 holds with $p=1$.

Now consider more general claims $\xi$. Recall that, if $K_{T}$ is a cone and $\xi \in K_{T}$ a.s., then $\Xi_{t}^{\xi} \subset \Xi_{t}^{0}$ for all $t$.

Theorem 4.3 If the solvency sets are proper and the acceptance sets are continuous from below, then (SNR) yields that $\hat{\Xi}_{t}^{\xi}$ is closed in probability for all $t$ and any $\xi \in \mathcal{L}^{p}\left(\mathbb{R}^{d}, \mathcal{F}_{T}\right)$, so that $\hat{\Xi}_{t}^{\xi}=\mathcal{L}^{0}\left(X_{t}^{\xi}, \mathcal{F}_{t}\right)$ for a random closed set $X_{t}^{\xi}, t=0, \ldots, T$.

Proof Assume that $k_{t}^{n}+\gamma_{t}^{n} \rightarrow \zeta_{t} \in \mathcal{L}^{0}\left(\mathbb{R}^{d}, \mathcal{F}_{t}\right)$ a.s. for $k_{t}^{n} \in \mathcal{L}^{0}\left(K_{t}, \mathcal{F}_{t}\right)$ and $\gamma_{t}^{n} \in M=\mathrm{R}_{t, t+1}\left(\Xi_{t+1}^{\xi}\right)$ such that $k_{t}^{n}+\gamma_{t}^{n} \in \hat{\Xi}_{t}^{0}$. Since $M$ is $\mathcal{L}^{0}$-closed and convex, we may assume by [24, Lemma 2.1.2] that $k_{t}^{n} \rightarrow k_{t} \in \mathcal{L}^{0}\left(K_{t}, \mathcal{F}_{t}\right)$ on the set $A=\left\{\liminf _{n}\left\|k_{t}^{n}\right\|<\infty\right\}$. Hence, $\gamma_{t}^{n} \rightarrow \gamma_{t} \in M$, so that $\zeta_{t}=k_{t}+\gamma_{t} \in \hat{\Xi}_{t}^{\xi}$.

If $\mathrm{P}(\Omega \backslash A)>0$, assume that $k_{t}^{n}=\gamma_{t}^{n}=\zeta_{t}=0$ on $A$ by $\mathcal{F}_{t^{-}}$-decomposability, and use the normalisation procedure, i.e. obtain $\tilde{k}_{t}^{n}$ and $\tilde{\gamma}_{t}^{n}$ by scaling $k_{t}^{n}$ and $\gamma_{t}^{n}$ with $c_{t}^{n}=\left(1+\left\|k_{t}^{n}\right\|\right)^{-1}$. We may assume that $\left\|\tilde{\gamma}_{t}^{n}\right\| \leq 2$, since $c_{t}^{n} \zeta_{t} \rightarrow 0$, so that $\tilde{k}_{t}^{n}+\tilde{\gamma}_{t}^{n} \rightarrow 0$. Arguing as previously, $\tilde{k}_{t}^{n} \rightarrow \tilde{k}_{t} \in \mathcal{L}^{0}\left(K_{t}, \mathcal{F}_{t}\right)$ in $\mathcal{L}^{p}$, and $\left\|\tilde{k}_{t}\right\|=1$ on $\Omega \backslash A$. Therefore, $\tilde{\gamma}_{t}^{n} \rightarrow \tilde{\gamma}_{t}=-\tilde{k}_{t}$ in $\mathcal{L}^{p}$, so that $\tilde{k}_{t}+\tilde{\gamma}_{t}=0$. Notice that

$$
M=\operatorname{cl}_{0}\left(\left(-\mathrm{A}_{t+1, T}+\xi\right) \cap \mathcal{L}^{0}\left(\mathbb{R}^{d}, \mathcal{F}_{t}\right)\right)
$$

By convexity,

$$
\tilde{\gamma}_{t}^{n} \in \operatorname{cl}_{0}\left(-\mathrm{A}_{t+1, T}+c_{t}^{n} \xi\right) \cap \mathcal{L}^{0}\left(\mathbb{R}^{d}, \mathcal{F}_{t}\right) .
$$

Since $\left\|\tilde{\gamma}_{t}^{n}\right\| \leq 2$, assume without loss of generality that $\tilde{\gamma}_{t}^{n} \in \operatorname{cl}_{p}\left(-\mathrm{A}_{t+1, T}+c_{t}^{n} \xi\right)$. To see this, it suffices to approximate the sequence $\tilde{\gamma}_{t}^{n}$ by $\bar{\gamma}_{t}^{m n} \in\left(-\mathrm{A}_{t+1, T}+\right.$ $\left.c_{t}^{n} \xi\right), m \geq 1$, and multiply the latter by $\mathbf{1}_{\left\|\bar{\gamma}_{t}^{m n}\right\| \leq 3}$. Letting $n \rightarrow \infty$, 4.6) yields that

$$
-\tilde{\gamma}_{t} \in \overline{\mathrm{A}}_{t, T}^{p} \cap \mathcal{L}^{0}\left(K_{t}, \mathcal{F}_{t}\right)=\{0\} .
$$

Thus, $\gamma_{t}=0$, so that $\mathrm{P}(\Omega \backslash A)=0$ and the conclusion follows.

Lemma 4.3 (NRA2) is equivalent to

$$
\Xi_{t}^{\xi} \subset \mathcal{L}^{0}\left(K_{t}, \mathcal{F}_{t}\right)+\mathcal{C}_{t-1, t}, \quad t=0, \ldots, T,
$$

for any $\xi \in \mathcal{L}^{0}\left(K_{T}, \mathcal{F}_{T}\right)$. If (4.4) holds, then (NRA2) implies (NARA). 
Proof Note that $\left(\eta_{t}+\mathrm{A}_{t, T}\right)$ intersects $\mathcal{L}^{0}\left(K_{T}, \mathcal{F}_{T}\right)$ if and only if

$$
\eta_{t} \in\left(-\mathrm{A}_{t, T}+\mathcal{L}^{0}\left(K_{T}, \mathcal{F}_{T}\right)\right) \cap \mathcal{L}^{0}\left(\mathbb{R}^{d}, \mathcal{F}_{t}\right),
$$

equivalently, $\eta_{t} \in \Xi_{t}^{\xi}$ for some $\xi=k_{T} \in \mathcal{L}^{0}\left(K_{T}, \mathcal{F}_{T}\right)$.

Denote by $K_{t}^{*}=\left\{x:\langle x, u\rangle \geq 0, u \in K_{t}\right\}$ the positive dual set to $K_{t}$ and assume that $K_{t}^{*} \backslash\{0\}$ is a subset of the interior of $\mathbb{R}_{+}^{d}$ for all $t$.

Definition 4.2 For $t \leq T$, an adapted process $Z=\left(Z_{s}\right)_{s=t, \ldots, T}$ is a $q$ integrable $t$-weakly consistent price system if it is a $\mathrm{Q}$-martingale for $\mathrm{Q} \sim \mathrm{P}$ such that $Z_{s}$ is a $q$-integrable under $\mathrm{Q}, \mathcal{F}_{s}$-measurable selection of $K_{s}^{*}$ for every $s \geq t$ and $Z_{t} \neq 0$ a.s. We denote by $\mathcal{M}_{t, T}^{q, w}(\mathrm{Q})$ the set all $q$-integrable $t$-weakly consistent price systems under $\mathrm{Q}$, where $q \in[1, \infty]$.

The following result characterises the prices under (SNR) and (NARA) conditions and so may be viewed as the Fundamental Theorem of Asset Pricing in our framework.

Theorem 4.4 Assume the coherent conical setting and that the solvency sets are continuous from below. Let $q$ be the conjugate of the number $p$ that stems from the definition of the acceptance sets.

(i) (NARA) is equivalent to the fact that, for each $t$, there exists $Z \in$ $\mathcal{M}_{t, T}^{q, w}(\mathrm{P})$ such that

$$
\mathrm{E}\left\langle Z_{u}, \eta_{u}\right\rangle \geq 0 \text { for all } \eta_{u} \in \mathcal{C}_{u-1, u}, u=t+1, \ldots, T .
$$

(ii) If $\operatorname{int} K_{t}^{*} \neq \emptyset$ a.s. for all $t$, then (SNR) is equivalent to the fact that, for each $t$, there exists $Z \in \mathcal{M}_{t, T}^{q, w}(\mathrm{P})$ such that (4.10) holds and $Z_{t} \in$ $\mathcal{L}^{0}\left(\operatorname{int} K_{t}^{*}, \mathcal{F}_{t}\right)$

Proof (i) The existence of $Z \in \mathcal{M}_{t, T}^{q, w}(\mathrm{P})$ such that $\mathrm{E}\left\langle Z_{t}, \eta\right\rangle \geq 0$ for all $\eta \in$ $\mathcal{C}_{t, t+1}+\cdots+\mathcal{C}_{T-1, T}$ is a direct consequence of the Hahn-Banach separation theorem and Theorem 4.2(i), since we may take $p=1$.

To prove the converse implication, for each $t$, assume the existence of $Z \in$ $\mathcal{M}_{t, T}^{q, w}(\mathrm{P})$ and consider $x_{T} \in \mathrm{A}_{t, T}^{p}$. Then

$$
x_{T}=-k_{t}-\left(k_{t+1}+\eta_{t+1}\right)-\cdots-\left(k_{T}+\eta_{T}\right),
$$

where $\eta_{s} \in \mathcal{C}_{s-1, s}$ and $k_{s} \in \mathcal{L}^{0}\left(K_{s}, \mathcal{F}_{s}\right)$ for $s \geq t$. Since $\eta_{s}=\eta_{s}^{\prime}+\eta_{s}^{\prime \prime}$ with $\eta_{s}^{\prime} \in \mathcal{C}_{s-1, s} \cap \mathcal{L}_{s-1, s}^{p}$ and $\eta_{s}^{\prime \prime} \in \mathcal{L}^{0}\left(\mathbb{R}_{+}^{d}, \mathcal{F}_{s}\right)$, we may merge $\eta_{s}^{\prime \prime}$ and $k_{s}$ and suppose without loss of generality that $\eta_{s}=\eta_{s}^{\prime}$.

Using the backward induction on $t \leq T$, we show that $\mathrm{E}\left\langle Z_{T}, x_{T}\right\rangle \leq 0$. If $x_{T}=-k_{T-1}-k_{T}-\eta_{T}$, this is trivial. Since $\eta_{t+1}, k_{t} \in \mathcal{L}_{t, t+1}^{p}$, there exists a partition $\left(B_{t}^{i}\right)_{i \geq 1}$ from $\mathcal{F}_{t}$ such that $\eta_{t+1} \mathbf{1}_{B_{t}^{i}}, k_{t} \mathbf{1}_{B_{t}^{i}} \in \mathcal{L}^{p}\left(\mathbb{R}^{d}, \mathcal{F}_{t}\right)$ for all $i \geq 1$. Then,

$$
x_{t+1}^{i}=\left(-k_{t+1}-\cdots-k_{T}-\eta_{t+2}-\cdots-\eta_{T}\right) \mathbf{1}_{B_{t}^{i}} \in \mathrm{A}_{t+1, T}^{p}, \quad i \geq 1 .
$$


Moreover,

$$
\begin{aligned}
\mathrm{E}\left\langle Z_{T}, x_{T}\right\rangle & =\sum_{i=1}^{\infty} \mathrm{E}\left\langle Z_{T}, x_{t+1}^{i}\right\rangle+\sum_{i=1}^{\infty} \mathrm{E}\left\langle Z_{T},-k_{t} \mathbf{1}_{B_{t}^{i}}\right\rangle+\mathrm{E}\left\langle Z_{t},-\eta_{t+1}\right\rangle \mathbf{1}_{B_{t}^{i}}, \\
& =\sum_{i=1}^{\infty} \mathrm{E}\left\langle Z_{T}, x_{t+1}^{i}\right\rangle+\sum_{i=1}^{\infty} \mathrm{E}\left\langle Z_{t},-k_{t} \mathbf{1}_{B_{t}^{i}}\right\rangle+\mathrm{E}\left\langle Z_{t+1},-\eta_{t+1} \mathbf{1}_{B_{t}^{i}}\right\rangle \\
& \leq \sum_{i=1}^{\infty} \mathrm{E}\left\langle Z_{T}, x_{t+1}^{i}\right\rangle .
\end{aligned}
$$

The induction hypothesis yields that $\mathrm{E}\left\langle Z_{T}, x_{t+1}^{i}\right\rangle \leq 0$. Hence, $\mathrm{E}\left\langle Z_{T}, x_{T}\right\rangle \leq 0$. Therefore, $\mathrm{E}\left\langle Z_{T}, x_{T}\right\rangle \leq 0$ for all $x_{T} \in \overline{\mathrm{A}}_{t, T}^{p}$. In particular, if $x_{T}=x_{t} \in$ $\mathcal{L}^{0}\left(\mathbb{R}_{+}^{d}, \mathcal{F}_{t}\right)$, then $\mathrm{E}\left\langle Z_{t}, x_{t}\right\rangle \leq 0$ and finally $\mathrm{E}\left\langle Z_{t}, x_{t}\right\rangle=0$. Since $Z_{t} \in \operatorname{int} \mathbb{R}_{+}^{d}$, we have $x_{T}=0$, i.e. (NARA) holds by Theorem 4.2 (i).

(ii) Replicate the proof of (i) using the Hahn-Banach theorem and following the arguments of [25, Th. 4.1] in order to construct $Z \in \mathcal{M}_{t, T}^{q, w}(\mathrm{P})$ such that $Z_{t} \in \mathcal{L}^{0}\left(\operatorname{int} K_{t}^{*}, \mathcal{F}_{t}\right)$.

Remark 1 Condition (4.10) can be equivalently written in terms of the conditional expectation as $\mathrm{E}\left(\left\langle Z_{u}, \eta_{u}\right\rangle \mid \mathcal{F}_{u-1}\right) \geq 0$ a.s., which also corresponds to the duality pairing in modules, see 15. Indeed, suppose that (4.10) holds. Then $\mathrm{E}\left(\left\langle Z_{u}, \eta_{u} \mathbf{1}_{A_{u-1}}\right\rangle\right) \geq 0$ for all $A_{u-1} \in \mathcal{F}_{u-1}$. Therefore, $\mathrm{E}\left(\left\langle Z_{u}, \eta_{u}\right\rangle \mid \mathcal{F}_{u-1}\right) \geq 0$. The opposite implication is obvious. In other words, (4.10) means that $Z_{u}$ belongs to the positive dual of $\mathcal{C}_{u-1, u}$.

If the acceptance sets with $p=\infty$ are generated by convex families $\mathcal{Z}_{t, s}$ (see Example 4), then (4.10) means that $Z_{t}$ belongs to the closure of $\mathcal{Z}_{t, s}$ with respect to the $\mathcal{F}_{t}$-bounded convergence in probability. If $K_{t}$ are all half-spaces (in the frictionless setting), then (NARA) is equivalent to the existence of a martingale $Z_{u}=\phi_{u} S_{u}$, where $S_{u}$ is the price vector, such that (4.10) holds.

\section{Good Deals hedging}

Assume that $\mathcal{C}_{t, s}$ consists of random vectors $\left(\eta_{s}, 0, \ldots, 0\right) \in \mathcal{L}^{p}\left(\mathbb{R}^{d}, \mathcal{F}_{s}\right)$ with all vanishing components except the first one and such that $r_{t, s}\left(\eta_{s}\right) \leq 0$ for a univariate dynamic risk measure $r_{t, s}$. This corresponds to the case, when the acceptability at each step is assessed by calculating the risk of a portfolio expressed in the units of the first asset, most importantly, cash. An arbitrage opportunity in this setting is called a Good Deal, see [7.

For simplicity, consider the one period setting with zero interest rate and two assets exchangeable without transaction costs, so that without loss of generality the first asset is assumed to be cash and the second one is a risky asset priced at $S_{t}$ for $t=0,1$. Let $\xi$ be the cash value of a terminal claim. If $x_{0}$ is the initial endowment (in cash), then the terminal value of a portfolio is

$$
V_{1}=\left(x_{0}, 0\right)+\left(-k_{0} S_{0}, k_{0}\right)+\left(-k_{1} S_{1}, k_{1}\right)-\left(\eta^{\prime}, \eta^{\prime \prime}\right),
$$


where $\eta^{\prime}$ and $\eta^{\prime \prime}$ are acceptable with respect to some static convex risk measure $r$, meaning that $r\left(\eta^{\prime}\right) \leq 0$ and $r\left(\eta^{\prime \prime}\right) \leq 0$. Given the choice of the acceptance set $\mathcal{C}_{0,1}$, we have $\eta^{\prime \prime}=0$, so that $V_{1}$ suffices to pay the claim if

$$
x_{0}+k_{0}\left(S_{1}-S_{0}\right)-\eta^{\prime} \geq \xi .
$$

The smallest value of $x_{0}$ that ensures the existence of an acceptable $\eta^{\prime}=$ $-\xi+x_{0}+k_{0}\left(S_{1}-S_{0}\right)$, satisfying the above inequality, equals the infimum of $r\left(k_{0}\left(S_{1}-S_{0}\right)-\xi\right)$ over all deterministic $k_{0} \in \mathbb{R}$. For instance, the zero claim $\xi=0$ can be hedged with a negative initial capital if $r\left(S_{1}-S_{0}\right)<0$ or $r\left(S_{0}-S_{1}\right)<0$, and this means the existence of a Good Deal arbitrage. If this is the case and the risk measure is coherent, then also

$$
r\left(k_{0}\left(S_{1}-S_{0}\right)-\xi\right) \leq r\left(k_{0}\left(S_{1}-S_{0}\right)\right)+r(-\xi)<0
$$

for sufficiently large positive $k_{0}$ if $r\left(S_{1}-S_{0}\right)<0$ (or negative $k_{0}$ if $r\left(S_{0}-S_{1}\right)<$ 0 ), meaning that any claim with finite $r(-\xi)$ can be also hedged with a negative initial investment. In other words, the No Good Deals (NGD) Arbitrage condition becomes

$$
r\left(S_{1} / S_{0}\right) \geq-1 \quad \text { and } \quad r\left(-S_{1} / S_{0}\right) \geq 1 .
$$

Our setting is more general as the Good Deals hedging, since it allows for more general acceptance sets and eliminates the prescribed choice of the single asset in order to assess the acceptability. As a result, the no arbitrage conditions become stronger and the superhedging price declines. To illustrate this, consider the above two assets one period setting with the acceptance set $\mathcal{C}_{0,1}$ that consists of all $\left(\eta^{\prime}, \eta^{\prime \prime}\right)$ such that $r\left(\eta^{\prime}\right) \leq 0$ and $r\left(\eta^{\prime \prime}\right) \leq 0$. By allowing a non-trivial $\eta^{\prime \prime}$, it is possible to decrease the price of a terminal cash claim $\xi$. For this, note $\xi$ can be paid if

$$
\begin{cases}x_{0}-k_{0} S_{0}-k_{1} S_{1}-\eta^{\prime} & \geq \xi \\ k_{0}+k_{1}-\eta^{\prime \prime} & \geq 0\end{cases}
$$

for some deterministic $k_{0}, \mathcal{F}_{1}$-measurable $k_{1}$, and acceptable $\eta^{\prime}, \eta^{\prime \prime}$. This increases the hedging possibilities and so leads to a decrease of the superhedging price, but also creates extra arbitrage opportunities. In particular, considering $\left(\eta^{\prime} \cdot \eta^{\prime \prime}\right) \in \mathcal{C}_{0,1}$ with $\eta^{\prime}=0$, the arbitrage becomes possible if $r\left(\left(k_{0}\left(S_{1}-S_{0}\right)+x_{0}\right) / S_{1}\right) \leq 0$ for some $x_{0}<0$ and $K_{0} \in \mathbb{R}$. By letting $x_{0}$ increase to zero, we see that the necessary no arbitrage condition in addition to (5.1) yields that

$$
r\left(S_{0} / S_{1}\right) \geq-1 \quad \text { and } \quad r\left(-S_{0} / S_{1}\right) \geq 1 .
$$

It corresponds to the fact that in two assets, the position expressed in one of them may be not acceptable, while the position expressed in the other one may be acceptable. The necessary and sufficient no arbitrage condition is stronger and should also include all possible combinations of the two assets. 
Assume that $\xi=\left(S_{1}-K\right)^{+}$for some $K>0$ and that the support of $S_{1}$ is

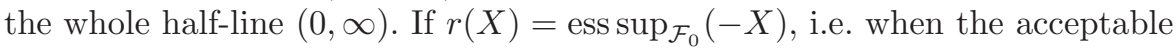
positions are non-negative random variables, then the minimal price

$$
x_{0}=\inf _{k_{0} \in \mathbb{R}} r\left(k_{0}\left(S_{1}-S_{0}\right)-\xi\right)
$$

equals $S_{0}$. If $r$ is non-trivial, we have $x_{0} \leq S_{0}+r\left(S_{1}-\xi\right)$. Note that $S_{1}-$ $\xi=S_{1} \wedge K$, so that $x_{0} \leq S_{0}+r\left(S_{1}\right) \wedge K$ and, finally, $x_{0} \leq S_{0}-r\left(S_{1}\right)$, where $r\left(S_{1}\right)<0$ given that $S_{1}>0$ and $r$ is non-trivial. This simple example illustrates the decrease of the super-hedging price in presence of a non-trivial risk measure.

Example 7 Assume that the risk measure $r$ is the negative essential infimum, that is, consider the setting of conditional cores from Section 6 . Then the NGD arbitrage is not possible if $\operatorname{ess}_{\inf } S_{\mathcal{F}_{0}} S_{1} \leq S_{0} \leq \operatorname{ess}_{\sup _{\mathcal{F}_{0}}} S_{1}$. With this choice of the risk measure, the NGD condition coincides with the (SNR) condition, see Theorem 8.1

\section{Conditional core as risk measure}

Assume that $p=\infty$ and $\mathcal{C}_{t, s}=\mathcal{L}^{0}\left(\mathbb{R}_{+}^{d}, \mathcal{F}_{s}\right)$ for all $0 \leq t \leq s \leq T$, so that $\mathrm{R}_{t, s}^{0}(\Xi)=\Xi \cap \mathcal{L}^{0}\left(\mathbb{R}^{d}, \mathcal{F}_{t}\right)$ for any upper set $\Xi \subset \mathcal{L}^{0}\left(\mathbb{R}^{d}, \mathcal{F}_{s}\right)$. If $X$ is an upper random closed set, then

$$
\mathrm{R}_{t, s}^{0}(X)=\mathrm{R}_{t, s}(X)=\mathbf{m}\left(X \mid \mathcal{F}_{t}\right)
$$

where the latter notation designates the largest $\mathcal{F}_{t}$-measurable random closed subset of $X$, called the conditional core of $X$, see [26, Def. 4.1]. An acceptable portfolio process is characterised by $V_{t-1}-V_{t} \in K_{t}$ a.s. for $t=1, \ldots, T$. Then $\mathrm{A}_{t, s}$ becomes the sum of $\mathcal{L}^{0}\left(-K_{u}, \mathcal{F}_{u}\right)$ for $u=t, \ldots, s$, exactly like in the classical theory of markets with transaction costs [24]. For claim $\xi$, the set $\Xi_{t}^{\xi}$ defined in Section 3 becomes the set of superhedging prices that was used in 27] to define a risk measure of $\xi$.

The classical no arbitrage condition $\left(\mathrm{NA}^{\mathrm{s}}\right.$ ) (no strict arbitrage opportunity at any time, see [24, Sec. 3.1.4]) then becomes (4.2); (SNA) (strong no arbitrage, see Condition (iii) in [24, Sec. 3.2.2]) then becomes the special case of (SNAR).

Theorem 6.1 Suppose that the solvency sets $\left(K_{t}\right)_{t=0, \ldots, T}$ are strictly proper. Then $(\mathrm{SNR}),\left(\mathrm{NA}^{\mathrm{s}}\right)$ and $(\mathrm{SNA})$ are all equivalent and are also equivalent to each of the following conditions.

(i) $\overline{\mathrm{A}}_{t, T}^{p} \cap \mathcal{L}^{0}\left(K_{t}, \mathcal{F}_{t}\right)=\{0\}$, for all $t \leq T-1$.

(ii) $\mathrm{A}_{t, T}$ is closed in $\mathcal{L}^{0}$ and $\mathrm{A}_{t, T} \cap \mathcal{L}^{0}\left(K_{t}, \mathcal{F}_{t}\right)=\{0\}$, for all $t \leq T-1$. 
Proof (SNR) is equivalent to (i) by Theorem 4.2(ii). The equivalence of $\left(\mathrm{NA}^{\mathrm{S}}\right)$ and (SNA) follows from Lemma 4.2 given that (4.4) trivially holds.

The implication (i) $\Rightarrow$ (ii) is simple to show by induction. First, $A_{T, T}$ is closed. Assume that $-k_{t}^{n}-\cdots-k_{T}^{n} \rightarrow \xi$ a.s. for $k_{u}^{n} \in \mathcal{L}^{0}\left(K_{u}, \mathcal{F}_{u}\right), u \geq t$. On the set $\left\{\liminf _{n}\left\|k_{t}^{n}\right\|=\infty\right\}$, we use the normalisation procedure to arrive at a contradiction with (i). Otherwise, suppose that $-k_{t}^{n} \rightarrow-k_{t} \in-K_{t}$, so that we may use the induction hypothesis to conclude.

In order to derive the closedness of $A_{t, r}$ under (SNA), it suffices to follow the proof of [24, Lemma 3.2.8]. Indeed, since $K_{t}^{0}$ is a linear space, the recession cone

$$
K_{t}^{\infty}=\bigcap_{\alpha>0} \alpha K_{t}=\left\{x \in \mathbb{R}^{d}: K_{t}+\alpha x \subset K_{t} \forall \alpha>0\right\}
$$

satisfies $K_{t}^{0} \subset K_{t}^{\infty}$, see 30]. Therefore, $k_{t}+\alpha x \in K_{t}$ for all $k_{t} \in K_{t}, x \in K_{t}^{0}$, and all $\alpha \in \mathbb{R}$. Furthermore, (SNA) trivially implies $\mathrm{A}_{t, T} \cap \mathcal{L}^{0}\left(K_{t}, \mathcal{F}_{t}\right)=\{0\}$ for all $t$.

In order to show that (ii) implies $\left(\mathrm{NA}^{\mathrm{s}}\right)$, assume

$$
-k_{0}-\cdots-k_{t}=\tilde{k}_{t} \in \mathrm{A}_{0, t} \cap \mathcal{L}^{0}\left(K_{t}, \mathcal{F}_{t}\right) .
$$

Then $k_{0} \in \mathrm{A}_{0, T} \cap \mathcal{L}^{0}\left(K_{0}, \mathcal{F}_{0}\right)$, i.e. $k_{0}=0$ by (ii). Similarly, $k_{1}=\cdots=k_{t-1}=0$, so that $-k_{t}=\tilde{k}_{t}=0$, since $K_{t}$ is strictly proper. Thus, $\left(\mathrm{NA}^{\mathrm{s}}\right)$ holds.

At last, $\left(\mathrm{NA}^{\mathrm{s}}\right)$ yields $(\mathrm{SNA})$, so that $\mathrm{A}_{t, T}$ is closed in $\mathcal{L}^{0}$. Finally, (SNA) yields (4.3) and so $\mathrm{A}_{t, T} \cap \mathcal{L}^{0}\left(K_{t}, \mathcal{F}_{t}\right)=\{0\}$, that is, (i) holds.

(NA2) (no arbitrage opportunity of the second kind) from [31] and 24, p. 135] has the same formulation as (NRA2).

Lemma 6.1 Assume that the solvency sets are cones.

(i) (NA2) is equivalent to $\Xi_{t}^{0}=\mathcal{L}^{0}\left(K_{t}, \mathcal{F}_{t}\right)$ for all $t \leq T$.

(ii) (NA2) is equivalent to

$$
\mathbf{m}\left(K_{t} \mid \mathcal{F}_{t-1}\right) \subset K_{t-1}, \quad t=1, \ldots, T .
$$

(iii) If the solvency sets are strictly proper, then (NA2) implies (SNR).

Proof (i) By Lemma 4.3 and Lemma 3.1(iv), $\Xi_{t}^{0}=\mathcal{L}^{0}\left(K_{t}, \mathcal{F}_{t}\right)$ yields (4.9), and so implies (NA2). In the other direction, (NA2) yields that $\Xi_{t}^{0} \subset \mathcal{L}^{0}\left(K_{t}, \mathcal{F}_{t}\right)+$ $\mathcal{C}_{t-1, t}$, while (3.3) and the choice of $\mathcal{C}_{t-1, t}$ yields that $\Xi_{t}^{0} \supset \mathcal{L}^{0}\left(K_{t}, \mathcal{F}_{t}\right)$.

(ii) If $\Xi_{t}^{0} \subset \mathcal{L}^{0}\left(K_{t}, \mathcal{F}_{t}\right)$, then

$$
\Xi_{t+1}^{0} \cap \mathcal{L}^{0}\left(\mathbb{R}^{d}, \mathcal{F}_{t}\right) \subset \mathcal{L}^{0}\left(K_{t}, \mathcal{F}_{t}\right)
$$

for all $t$ by (3.3). Since $\mathcal{L}^{0}\left(K_{t+1}, \mathcal{F}_{t+1}\right) \subset \Xi_{t+1}^{0}$, we obtain (6.1).

If (6.1) holds, then

$$
\Xi_{T-1}^{0}=\mathcal{L}^{0}\left(K_{T-1}, \mathcal{F}_{T-1}\right)+\mathbf{m}\left(K_{T} \mid \mathcal{F}_{T-1}\right) \subset \mathcal{L}^{0}\left(K_{T-1}, \mathcal{F}_{T-1}\right) .
$$


Assume that $\Xi_{s}^{0} \subset \mathcal{L}^{0}\left(K_{s}, \mathcal{F}_{s}\right)$ for $s=t+1, \ldots, T$. Then

$$
\begin{aligned}
\Xi_{t}^{0} & =\mathcal{L}^{0}\left(K_{t}, \mathcal{F}_{t}\right)+\left(\Xi_{t+1}^{0} \cap \mathcal{L}^{0}\left(\mathbb{R}^{d}, \mathcal{F}_{t}\right)\right) \\
& \subset \mathcal{L}^{0}\left(K_{t}, \mathcal{F}_{t}\right)+\mathbf{m}\left(K_{t+1} \mid \mathcal{F}_{t}\right) \subset \mathcal{L}^{0}\left(K_{t}, \mathcal{F}_{t}\right)
\end{aligned}
$$

The proof is finished by the induction argument.

(iii) Since $\Xi_{t}^{0}$ is closed in probability under (SNR), we have $\Xi_{t}^{0}=\hat{\Xi}_{t}^{0}$, and (SNR) yields that $\mathcal{L}^{0}\left(K_{t}, \mathcal{F}_{t}\right) \cap \mathcal{L}^{0}\left(-K_{t}, \mathcal{F}_{t}\right)=\{0\}$, which is the case if the solvency sets are strictly proper.

For conical solvency sets satisfying $\mathbf{m}\left(K_{t}^{0} \mid \mathcal{F}_{t-1}\right) \subset K_{t-1}^{0}, t \leq T$, in particular, for strictly proper ones, $\left(\mathrm{NA}^{\mathrm{s}}\right)$ is equivalent to the existence of a $\mathrm{Q}$ martingale evolving in the relative interiors of $\left(K_{t}^{*}\right)_{t=0, \ldots, T}$ for a probability measure $\mathrm{Q}$ equivalent to $\mathrm{P}$, see [24, Th. 3.2.2]. Such a martingale is called a strictly consistent price system. If int $K_{t}^{*} \neq \emptyset$ for all $t$, this result follows from Theorem 4.4(ii).

Note that $\Xi_{T}^{\xi}=\mathcal{L}^{0}\left(X_{T}^{\xi}, \mathcal{F}_{T}\right)$ for $X_{T}=\xi+K_{T}$, and $\Xi_{T-1}^{\xi}=\mathcal{L}^{0}\left(X_{T-1}^{\xi}, \mathcal{F}_{T-1}\right)$ is the family of selections for a (possibly non-closed) random set $X_{T-1}^{\xi}=$ $K_{T-1}+\mathbf{m}\left(X_{T}^{\xi} \mid \mathcal{F}_{T-1}\right)$. One needs additional assumptions of the no arbitrage type in order to extend this interpretation for $\Xi_{t}^{\xi}$ with $t \leq T-2$. Precisely the sum above should be closed, so that $\mathbf{m}\left(X_{t}^{\xi} \mid \mathcal{F}_{t-1}\right)$ exists for $t \leq T-1$, which makes it possible to apply Lemma 9.1

Theorem 6.2 Assume that the solvency sets are strictly proper and $\left(\mathrm{NA}^{\mathrm{s}}\right)$ (equivalently, (SNR) or (SNA)) holds. Then $\Xi_{t}^{\xi}=\mathcal{L}^{0}\left(X_{t}^{\xi}, \mathcal{F}_{t}\right)$, where $X_{t}^{\xi}$ is an $\mathcal{F}_{t}$-measurable random closed convex set, $t=0, \ldots, T$, such that $X_{T}^{\xi}=\xi+K_{T}$, and

$$
X_{t}^{\xi}=K_{t}+\mathbf{m}\left(X_{t+1}^{\xi} \mid \mathcal{F}_{t}\right), \quad t=T-1, \ldots, 0 .
$$

Proof It suffices to confirm the statement for $t=T-1$ and then use the induction. Indeed, by Theorem 6.1. $\left(\mathrm{NA}^{\mathrm{s}}\right)$ is equivalent to (SNR) so that Theorem 4.3 applies. Since $\Xi_{t}^{\xi}$ is $\mathcal{F}_{t}$-decomposable, Theorem 9.1 yields the existence of an $\mathcal{F}_{T-1}$-measurable closed set $X_{T-1}^{\xi}$ such that $\Xi_{T-1}^{\xi}=\mathcal{L}^{0}\left(X_{T-1}^{\xi}, \mathcal{F}_{T-1}\right)$. Since $X_{T}^{\xi}$ is closed,

$$
\begin{aligned}
\Xi_{T-1}^{\xi} & =\mathcal{L}^{0}\left(K_{T-1}, \mathcal{F}_{T-1}\right)+\mathcal{L}^{0}\left(\mathbf{m}\left(X_{T}^{\xi} \mid \mathcal{F}_{T-1}\right), \mathcal{F}_{T-1}\right), \\
& =\mathcal{L}^{0}\left(K_{T-1}+\mathbf{m}\left(X_{T}^{\xi} \mid \mathcal{F}_{T-1}\right), \mathcal{F}_{T-1}\right) \\
& =\mathcal{L}^{0}\left(X_{T-1}^{\xi}, \mathcal{F}_{T-1}\right)
\end{aligned}
$$

where $X_{T-1}^{\xi}$ is a random set by Lemma 9.1

Proposition 6.1 Suppose that the solvency sets are strictly proper. Then $\left(\mathrm{NA}^{\mathrm{s}}\right)$ holds if and only if $\Xi_{t}^{0}=\mathcal{L}^{0}\left(X_{t}^{0}, \mathcal{F}_{t}\right)$ for random closed sets $\left(X_{t}^{0}\right)_{t=0, \ldots, T}$ 
such that $X_{t}^{0} \cap\left(-K_{t}\right)=\{0\}$ a.s. for all $t$. In the conical case, the latter condition is equivalent to $\operatorname{int}\left(X_{t}^{0}\right)^{*} \neq \emptyset$ for all $t$, and, under $\left(\mathrm{NA}^{\mathrm{s}}\right)$,

$$
\mathrm{A}_{t, T}=\sum_{s=t}^{T} \mathcal{L}^{0}\left(-X_{s}^{0}, \mathcal{F}_{s}\right), \quad 0 \leq t \leq T,
$$

where $X_{t}^{0}$ is a strictly proper random closed convex cone for all $t$.

Proof Assume $\left(\mathrm{NA}^{\mathrm{s}}\right)$, so that Theorem6.2 applies. Let $-g_{t} \in \mathcal{L}^{0}\left(X_{t}^{0} \cap\left(-K_{t}\right), \mathcal{F}_{t}\right)$. Then $g_{t} \in K_{t}$ a.s., and there exist $k_{u} \in \mathcal{L}^{0}\left(K_{u}, \mathcal{F}_{u}\right), u=t, \ldots, T$, and $\tilde{g}_{T} \in \mathcal{L}^{0}\left(K_{T}, \mathcal{F}_{T}\right)$, such that $-g_{t}-k_{t}-k_{t+1}-\cdots-k_{T}=\tilde{g}_{T}$. Since (SNA) holds, $g_{t}+k_{t}=0$, and $g_{t}=0$. The reverse implication is trivial. In the conical case, since $K_{t}+K_{t}=K_{t}$ for all $t \leq T$, (6.3) follows from the inclusions

$$
K_{t} \subset X_{t}^{0} \subset K_{t}+\cdots+K_{T}, \quad t \leq T .
$$

Note that $X_{T}^{0}=K_{T}$ is strictly proper by assumption. Since

$$
X_{t-1}^{0}=K_{t-1}+\mathbf{m}\left(X_{t}^{0} \mid \mathcal{F}_{t-1}\right) \subset K_{t-1}+X_{t}^{0},
$$

the induction argument yields that $X_{t}^{0} \subset K_{t}+\cdots+K_{T}$. Since (SNA) holds under $\left(\mathrm{NA}^{\mathrm{s}}\right), X_{t}^{0}$ is strictly proper for all $t$.

By [24. Lemma 5.1.2], $\left(\mathrm{NA}^{\mathrm{s}}\right)$ holds if and only if $\Xi_{t}^{0}$ is closed and $\Xi_{t}^{0}=$ $\mathcal{L}^{0}\left(X_{t}^{0}, \mathcal{F}_{t}\right)$ with

$$
\operatorname{int} K_{t}^{*} \cap \operatorname{int}\left(X_{t}^{0}\right)^{*}=\operatorname{int} K_{t}^{*} \cap \operatorname{int} \mathbf{m}\left(X_{t+1}^{0} \mid \mathcal{F}_{t}\right)^{*} \neq \emptyset, \quad t \leq T .
$$

Finally, observe that

$$
\operatorname{int} K_{t}^{*} \cap \operatorname{int} \mathbf{m}\left(X_{t+1}^{0} \mid \mathcal{F}_{t}\right)^{*}=\operatorname{int} K_{t}^{*} \cap \mathbf{m}\left(X_{t+1}^{0} \mid \mathcal{F}_{t}\right)^{*}=\operatorname{int}\left(X_{t}^{0}\right)^{*} .
$$

Equation (6.3) means that, in the superhedging problem, we may replace solvency sets $K_{t}$ with $X_{t}^{0}$. The solvency sets $\left(X_{t}^{0}\right)_{t=0, \ldots, T}$ satisfy (NA2) condition by Lemma 6.1, which is generally required to obtain a dual characterisation of the superhedging prices, see Condition $\mathbf{B}$ (equivalent to (NA2)) in [24, Sec. 3.6.3]. Therefore, $\left(\mathrm{NA}^{\mathrm{s}}\right)$ suffices for [24, Th. 3.6.3] to hold provided that we consider the consistent price systems associated to $\left(X_{t}^{0}\right)_{t=0, \ldots, T}$.

Now consider (NRA) and (NARA) condition for the chosen acceptance sets. Assume that the solvency sets are conical and satisfy $K_{t}^{*} \backslash\{0\} \subset \operatorname{int} \mathbb{R}_{+}^{d}$. Since $\hat{\Xi}_{t}^{0}=\operatorname{cl}_{0}\left(\hat{\Xi}_{t}^{0}\right)=\operatorname{cl}_{0}\left(\Xi_{t}^{0}\right)=\Xi_{t}^{0},(\mathrm{NRA})$ and (NARA) are equivalent by Proposition 4.1 Denote by $\mathrm{A}_{t, T}^{p}(\mathrm{Q})$ and $\overline{\mathrm{A}}_{t, T}^{p}(\mathrm{Q})$ for $p \in[1, \infty]$ the variants of $\mathrm{A}_{t, T}^{p}$ and $\overline{\mathrm{A}}_{t, T}^{p}$ when the reference probability measure is $\mathbf{Q}$.

Proposition 6.2 The following statements are equivalent.

(i) $\overline{\mathrm{A}}_{t, T}^{p}(\mathrm{Q}) \cap \mathcal{L}^{0}\left(\mathbb{R}_{+}^{d}, \mathcal{F}_{t}\right)=\{0\}$, for all $t \leq T-1, p \in[1, \infty)$ and $\mathrm{Q} \sim \mathrm{P}$.

(ii) $\mathcal{M}_{t, T}^{q, w}(\mathrm{Q}) \neq \emptyset$ for every $t \leq T-1, \mathrm{Q} \sim \mathrm{P}$ and $p \in[1, \infty)$.

(iii) (NARA).

(iv) $\mathcal{M}_{t, T}^{\infty, w}(\mathrm{P}) \neq \emptyset$ for every $t \leq T-1$. 
(v) $\mathcal{M}_{t, T}^{1, w}(\mathrm{P}) \neq \emptyset$ for every $t \leq T-1$.

Proof By Theorem 4.4 (v) and (iii) are equivalent. We deduce the equivalence of (ii) and (iv) by following the proof of [24, Lemma 3.2.4], which makes it possible to construct a (weakly)-consistent price system (see Definition 4.2) from any consistent price system in $\mathcal{L}^{1}$. In particular, (v) implies (iv) and, clearly, (iv) implies (v). Then (iii) implies (ii), i.e. (NARA) holds for $Q$ in place of $P$. By Theorem 4.2, (i) holds. At last, (i) implies (NARA) by Theorem 4.2

\section{Arbitrage with acceptable expectations}

Assume that $p=1$, and let $\mathcal{C}_{t, s} \cap \mathcal{L}_{t, s}^{1}$ be the set of all $\eta_{s} \in \mathcal{L}_{t, s}^{1}$ such that $\mathrm{E}^{g}\left(\eta_{s} \mid \mathcal{F}_{t}\right)$ has all non-negative components. In other words, the acceptable positions are those having non-negative generalised conditional expectation. This is the weakest possible acceptability criterion, which is always the case (in the static setting) if the acceptance sets are dilatation monotonic.

The generalised conditional expectation is well defined for each $\gamma_{s} \in \overline{\mathcal{L}}_{t, s}^{1}$ by letting $\mathrm{E}^{g}\left(\gamma_{s} \mid \mathcal{F}_{t}\right)=\mathrm{E}^{g}\left(\gamma_{s}^{\prime} \mid \mathcal{F}_{t}\right)+\mathrm{E}\left(\gamma_{s}^{\prime \prime} \mid \mathcal{F}_{t}\right)$, where the expectation of $\gamma_{s}^{\prime \prime} \in$ $\mathcal{L}^{0}\left(\mathbb{R}_{+}^{d}, \mathcal{F}_{s}\right)$ may be infinite. If $X_{T}$ is an $\mathcal{F}_{T}$-measurable random upper convex set that admits at least one selection from $\mathcal{L}_{t, s}^{1}$, then let

$$
\mathrm{R}_{t, s}^{0}\left(X_{T}\right)=\left\{\mathrm{E}^{g}\left(\gamma_{s} \mid \mathcal{F}_{t}\right): \gamma_{s} \in \mathcal{L}_{\mathcal{F}_{t}}^{1}\left(X_{T}, \mathcal{F}_{s}\right)\right\},
$$

and $\mathrm{R}_{t, s}\left(X_{T}\right)=\mathrm{E}^{g}\left(X_{T} \mid \mathcal{F}_{t}\right)$ is the generalised conditional expectation of the random closed set $X_{T}$, see [26, Def. 6.3].

Let $\xi \in \mathcal{L}^{1}\left(\mathbb{R}^{d}, \mathcal{F}_{T}\right)$. By Lemma 3.1, $\hat{\Xi}_{T-1}^{\xi}$ is the family of selections of the (possibly, non-closed) random set

$$
X_{T-1}^{\xi}=K_{T-1}+\mathrm{E}\left(\xi \mid \mathcal{F}_{T-1}\right)+\mathrm{E}\left(K_{T} \mid \mathcal{F}_{T-1}\right),
$$

which is $\mathcal{F}_{T-1}$-measurable by Lemma 9.1 . Note that all solvency sets are integrable and so their generalised conditional expectation coincides with the usual one. Therefore,

$$
\begin{aligned}
\mathrm{R}_{T-2, T-1}\left(\hat{\Xi}_{T-1}^{\xi}\right) & =\mathrm{E}\left(X_{T-1}^{\xi} \mid \mathcal{F}_{T-2}\right) \\
& =\mathrm{E}\left(\xi \mid \mathcal{F}_{T-2}\right)+\mathrm{E}\left(K_{T-1}+\mathrm{E}\left(K_{t} \mid \mathcal{F}_{T-1}\right) \mid \mathcal{F}_{T-2}\right), \\
& =\mathrm{E}\left(\xi \mid \mathcal{F}_{T-2}\right)+\mathrm{E}\left(K_{T}+K_{T-1} \mid \mathcal{F}_{T-2}\right) .
\end{aligned}
$$

Since $k_{T} \in \mathcal{L}_{\mathcal{F}_{T-1}}^{1}\left(K_{T}, \mathcal{F}_{T}\right)$ and

$$
\begin{aligned}
& \mathrm{R}_{T-2, T-1}^{0}\left(\Xi_{T-1}^{\xi}\right) \\
& \quad=\left\{\mathrm{E}^{g}\left(k_{T-1}+\mathrm{E}^{g}\left(\xi+k_{T} \mid \mathcal{F}_{T-1}\right) \mid \mathcal{F}_{T-2}\right): k_{T-1} \in \mathcal{L}^{0}\left(K_{T-1}, \mathcal{F}_{T-1}\right)\right\}
\end{aligned}
$$

we deduce that $\mathrm{R}_{T-2, T-1}\left(\hat{\Xi}_{T-1}^{\xi}\right) \subset \mathrm{R}_{T-2, T-1}\left(\Xi_{T-1}^{\xi}\right)$. Since $\Xi_{T-1}^{\xi}$ is a subset of $\hat{\Xi}_{T-1}^{\xi}$, we have

$$
\mathrm{R}_{T-2, T-1}\left(\hat{\Xi}_{T-1}^{\xi}\right)=\mathrm{R}_{T-2, T-1}\left(\Xi_{T-1}^{\xi}\right) .
$$


Therefore,

$$
X_{T-2}^{\xi}=K_{T-2}+\mathrm{E}\left(\xi \mid \mathcal{F}_{T-2}\right)+\mathrm{E}\left(K_{T}+K_{T-1} \mid \mathcal{F}_{T-2}\right),
$$

Continuing recursively, we obtain $\hat{\Xi}_{t}^{\xi}=\mathcal{L}^{0}\left(X_{t}^{\xi}, \mathcal{F}_{t}\right)$ with a not necessarily closed $\mathcal{F}_{t}$-measurable random set

$$
X_{t}^{\xi}=K_{t}+\mathrm{E}\left(\xi \mid \mathcal{F}_{t}\right)+\mathrm{E}\left(K_{T}+K_{T-1}+\cdots+K_{t+1} \mid \mathcal{F}_{t}\right) .
$$

Notice that $X_{t}^{\xi}=X_{t}^{0}+\mathrm{E}\left(\xi \mid \mathcal{F}_{t}\right)$, i.e. $X_{t}^{0}$ determines all superhedging prices. Reformulating requirements from Definition 4.1, we arrive at the following result.

Proposition 7.1 For the risk arbitrage conditions formulated for the conditional expectation as the risk measure, the following hold.

(i) If the solvency sets are strictly proper, (SNR) is equivalent to

$$
\mathrm{E}\left(K_{t+1}+\cdots+K_{T} \mid \mathcal{F}_{t}\right) \cap\left(-K_{t}\right)=\{0\}, \text { a.s. }, \quad t=0, \ldots, T-1 .
$$

(ii) (NARA) is equivalent to

$$
\left(K_{t}+\mathrm{E}\left(K_{t+1}+\cdots+K_{T} \mid \mathcal{F}_{t}\right)\right) \cap \mathbb{R}_{-}^{d}=\{0\} \quad \text { a.s. }, \quad t=0, \ldots, T-1 .
$$

Note that statement (ii) above follows from (i). Theorem 4.4 yields the following result.

Proposition 7.2 Assume that the solvency sets $\left(K_{t}\right)_{t=0, \ldots, T}$ are cones. Then (NARA) (resp. (SNR)) is equivalent to the existence of a deterministic point $z \neq 0$ that belongs to all $K_{t}^{*}\left(\right.$ resp. $\left.\operatorname{int} K_{t}^{*}\right), t=0, \ldots, T$.

Proof Any acceptable position from $\mathcal{C}_{u-1, u}$ is of the form

$$
\eta_{u}=\left[\gamma_{u}-\mathrm{E}^{g}\left(\gamma_{u} \mid \mathcal{F}_{u-1}\right)\right]+\mathrm{E}^{g}\left(\gamma_{u} \mid \mathcal{F}_{u-1}\right)+\zeta_{u}^{+}
$$

with $\mathrm{E}^{g}\left(\gamma_{u} \mid \mathcal{F}_{u-1}\right) \in \mathbb{R}_{+}^{d}$ and $\zeta_{u}^{+} \in \mathcal{L}^{0}\left(\mathbb{R}_{+}^{d}, \mathcal{F}_{u}\right)$. Thus,

$$
\begin{aligned}
\mathrm{E}^{g}\left(\left\langle Z_{u}, \eta_{u}\right\rangle \mid \mathcal{F}_{u-1}\right) & \geq \mathrm{E}^{g}\left(\left\langle Z_{u}, \gamma_{u}-\mathrm{E}^{g}\left(\gamma_{u} \mid \mathcal{F}_{u-1}\right)\right| \mathcal{F}_{u-1}\right) \\
& =\mathrm{E}^{g}\left(\left\langle Z_{u}, \gamma_{u}\right\rangle \mid \mathcal{F}_{u-1}\right)-\left\langle Z_{u-1}, \mathrm{E}^{g}\left(\gamma_{u} \mid \mathcal{F}_{u-1}\right)\right\rangle .
\end{aligned}
$$

Hence, $\mathrm{E}^{g}\left(\left\langle Z_{u}, \eta_{u}\right\rangle \mid \mathcal{F}_{u-1}\right) \geq 0$ if there exists $Z \in \mathcal{M}_{t, T}^{\infty, w}(\mathrm{P})$, such that

$$
\mathrm{E}^{g}\left(\left\langle Z_{u}, \gamma_{u}\right\rangle \mid \mathcal{F}_{u-1}\right)=\left\langle Z_{u-1}, \mathrm{E}^{g}\left(\gamma_{u} \mid \mathcal{F}_{u-1}\right)\right\rangle \text { a.s. }
$$

for all $\gamma_{u} \in \mathcal{L}_{\mathcal{F}_{u-1}}^{1}\left(\mathbb{R}^{d}, \mathcal{F}_{u}\right)$. The equality follows from (4.10) by taking unconditional expectation (restricting to a partition if necessary) and applying the same reason with $-\gamma_{u}$. Given that $Z_{u}$ is essentially bounded, it is possible to let $\gamma_{u}$ be equal to one of the component of $Z_{u}$ multiplied by the corresponding basis vector. Thus, the square of every component of $\left(Z_{u}\right)_{u=0, \ldots, T}$ is a martingale, whence $Z_{u}$ equals to deterministic $z$ for all $u$.

The inverse implication follows from Theorem 4.4(i) applied to

$$
\eta_{u}=\gamma_{u}-\mathrm{E}^{g}\left(\gamma_{u} \mid \mathcal{F}_{u-1}\right) \in \mathcal{C}_{u-1, u} .
$$

The proof for (SNR) follows from the same argument and Theorem 4.4(ii). 
Remark 2 It is possible to derive the result of Proposition 7.2 from Proposition 7.1 by using the fact that the expectation of the cone $K_{t}$ is the whole space unless $K_{t}^{*}$ contains a deterministic point $z$ distinct from the origin and then $\mathrm{E} K_{t}$ is a subset of the half-space with outer normal $(-z)$. In order that $X_{t}^{0}$ does not intersect $\mathbb{R}_{-}^{d}$, all cones $K_{t}$ should have non-trivial expectation and the sum of these expectations has to be non-trivial. This amounts to the existence of a deterministic point $z \neq 0$ that belongs to $K_{0}^{*} \cap \cdots \cap K_{T}^{*}$.

\section{Application to the two-dimensional model}

Consider a financial market model composed of two assets. The first one has constant value 1 and the second one is a risky asset modelled by a bid-ask spread $Y_{t}=\left[S_{t}^{b}, S_{t}^{a}\right]$ such that $0<S_{t}^{b} \leq S_{t}^{a}$ a.s. for all $t \leq T$. This is Kabanov's model with the conical solvency set $K_{t}=C\left(Y_{t}\right)$, where $C\left(\left[s^{\prime}, s^{\prime \prime}\right]\right)$ is the positive dual to the smallest cone in $\mathbb{R}^{2}$ containing the set $\{1\} \times\left[s^{\prime}, s^{\prime \prime}\right]$.

Consider the acceptance sets from Section [6] so that the conditional core is the risk measure. Then $X_{T-1}^{0}$ is the sum of $K_{T-1}$ and $\mathbf{m}\left(X_{T}^{0}, \mathcal{F}_{T-1}\right)=$ $C\left(\mathbf{M}\left(Y_{T} \mid \mathcal{F}_{T-1}\right)\right)$, where $\left.\mathbf{M}\left(Y_{T} \mid \mathcal{F}_{T-1}\right)\right)$ is the conditional convex hull of $Y_{T}$, that is, the smallest $\mathcal{F}_{T-1}$-measurable random closed convex set that contains $Y_{T-1}$, see [26, Def. 5.1].

Since $X_{T-1}^{0}$ is a random closed set, iterating this argument yields that $X_{t}^{0}=C\left(\tilde{Y}_{t}\right)$ for $t=0, \ldots, T$, where $\tilde{Y}_{T}=Y_{T}$ and

$$
\tilde{Y}_{t}=\mathbf{M}\left(\tilde{Y}_{t+1} \mid \mathcal{F}_{t}\right) \cap Y_{t}, \quad t=T-1, \ldots, 0 .
$$

Note that we do not make any no arbitrage assumption to obtain $X_{t}^{0}$. Observe that $\tilde{Y}_{t}=\left[\tilde{S}_{t}^{b}, \tilde{S}_{t}^{a}\right]$, where $\tilde{S}_{T}^{a}=S_{T}^{a}, \tilde{S}_{T}^{b}=S_{T}^{b}$, and

$$
\tilde{S}_{t}^{a}=S_{t}^{a} \wedge \operatorname{ess}_{\sup _{\mathcal{F}_{t}}} \tilde{S}_{t+1}^{a}, \quad \tilde{S}_{t}^{b}=S_{t}^{b} \vee \operatorname{ess} \inf _{\mathcal{F}_{t}} \tilde{S}_{t+1}^{b}
$$

for $t=T-1, \ldots, 1$. Since $0<\tilde{S}_{t}^{b} \leq \tilde{S}_{t}^{a}$ a.s. for all $t$, (NRA) always holds. By Definition 4.1 and Lemma 4.3. we easily deduce the following result.

\section{Theorem 8.1}

(i) (SNR) holds if and only if $S_{t}^{b} \leq \operatorname{ess}_{\sup _{\mathcal{F}_{t}}} \tilde{S}_{t+1}^{a}$ and $S_{t}^{a} \geq \operatorname{essinf}_{\mathcal{F}_{t}} \tilde{S}_{t+1}^{b}$ a.s. with strict inequalities when $S_{t}^{b}<S_{t}^{a}$, for all $t \leq T-1$.

(ii) (NA2) holds if and only if $\operatorname{ess}_{\sup _{\mathcal{F}_{t}}} S_{t+1}^{a} \geq S_{t}^{a}$ and $S_{t}^{b} \geq \operatorname{essinf}_{\mathcal{F}_{t}} S_{t+1}^{b}$ a.s. for all $t \leq T-1$.

Remark $3\left(\mathrm{NA}^{\mathrm{s}}\right)$ is equivalent to $(\mathrm{SNR})$ in the proper case but also to the existence of a strictly consistent price system, see [24, Th. 3.2.2]. In the two asset case, the Grigoriev theorem, see [24, Th. 3.2.15] and [18, asserts that $\left(\mathrm{NA}^{\mathrm{s}}\right)$ is equivalent to the existence of a (possibly non-strict) consistent price system, i.e. the existence of a martingale $Z_{t}$ with respect to a probability measure $\mathrm{Q}$ equivalent to $\mathrm{P}$ such that $S_{t}^{b} \leq Z_{t} \leq S_{t}^{a}$ for all $t$. By Theorem [8.1. the existence of a consistent price system, equivalently, 
$\left(\mathrm{NA}^{\mathrm{s}}\right)$, implies $(\mathrm{SNR})$. Indeed, $\operatorname{ess}_{\sup _{\mathcal{F}_{t}}} \tilde{S}_{t+1}^{a} \geq \mathrm{E}_{\mathrm{Q}}\left(Z_{t+1} \mid \mathcal{F}_{t}\right) \geq S_{t}^{b}$ and similarly $\operatorname{essinf}_{\mathcal{F}_{t}} \tilde{S}_{t+1}^{b} \leq \mathrm{E}_{\mathrm{Q}}\left(Z_{t+1} \mid \mathcal{F}_{t}\right) \leq S_{t}^{a}$, the inequalities being strict when $S_{t}^{b}<S_{a}^{b}$.

Corollary 8.1 If there exist probability measures $\mathrm{Q}^{a}, \mathrm{Q}^{b}$ which are equivalent to $\mathrm{P}$, such that $S^{a}$ is a $\mathrm{Q}^{a}$-submartingale and $S^{b}$ is $a \mathrm{Q}^{b}$-supermartingale, then (NA2) holds.

The condition in the following corollary means that $\delta_{t}^{b}=S_{t}^{b} / S_{t-1}^{b}$ and $\delta_{t}^{a}=S_{t}^{a} / S_{t-1}^{a}$ admit conditional full supports on $\mathbb{R}_{+}$for all $t=1, \ldots, T$.

Corollary 8.2 If $\mathrm{P}\left(\delta_{t}^{b} \leq c \mid \mathcal{F}_{t-1}\right) \mathrm{P}\left(\delta_{t}^{a} \geq c \mid \mathcal{F}_{t-1}\right)>0$ a.s. for all $t=1, \ldots, T$ and all $c>0$, then (NA2) holds.

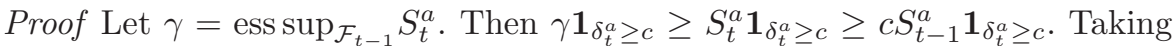
the conditional expectation yields that

$$
\gamma \mathrm{P}\left(\delta_{t}^{a} \geq c \mid \mathcal{F}_{t-1}\right) \geq c S_{t-1}^{a} \mathrm{P}\left(\delta_{t}^{a} \geq c \mid \mathcal{F}_{t-1}\right) .
$$

Then $\gamma \geq c S_{t-1}^{a}$, and letting $c \rightarrow \infty$ yields that $\gamma=+\infty$ a.s. Similarly, $\operatorname{ess}_{\inf } \mathcal{F}_{t-1} S_{t}^{b}=0$ a.s., and Theorem 8.1 (ii) applies.

Assume now that the acceptability criterion is based on the generalised conditional expectation as described in Section 7. By Proposition 7.2. (NARA) holds if and only if there is deterministic $z$ that belongs to all $Y_{t}, t=0, \ldots, T$, and (SNR) additionally requires that this point belongs to the interiors of $Y_{t}$.

Example 8 (Limit order book) Consider the two asset setting, where it is allowed to perform transactions up to one cash unit amount. This is a simple limit order book setting with only one break point. Then $K_{t}$ is the sum $\left[0, \alpha_{t}\right]+\left[0, \beta_{t}\right]+\mathbb{R}_{+}^{2}$, where $\alpha_{t}=\left(1,-S_{t}^{a}\right)$ and $\beta_{t}=\left(-1, S_{t}^{b}\right)$. By Proposition 7.1. (NARA) (with acceptability based on conditional expectation) holds if and only if

$$
\mathrm{E}\left[\sum_{s=t+1}^{T}\left(\left[0, \alpha_{s}\right]+\left[0, \beta_{s}\right]\right) \mid \mathcal{F}_{t}\right] \cap \mathbb{R}_{-}^{d}=\{0\}
$$

for all $t=0, \ldots, T-1$. The sum of segments $\left[0, \alpha_{s}\right]$ and $\left[0, \beta_{s}\right]$ is a random convex compact set called a zonotope. The setting can be easily extended to the case of limit order books with several break points.

\section{Appendix: Random sets and their selections}

Let $\mathbb{R}^{d}$ be the Euclidean space with norm $\|\cdot\|$ and the Borel $\sigma$-algebra $\mathcal{B}\left(\mathbb{R}^{d}\right)$. The closure of a set $A \subset \mathbb{R}^{d}$ is denoted by $\operatorname{cl} A$. A set-valued function $\omega \mapsto X(\omega)$ from a complete probability space $(\Omega, \mathcal{F}, \mathrm{P})$ to the family of all subsets of $\mathbb{R}^{d}$ is called $\mathcal{F}$-measurable (or graph-measurable) if its graph

$$
\operatorname{Gr} X=\left\{(\omega, x) \in \Omega \times \mathbb{R}^{d}: x \in X(\omega)\right\} \subset \Omega \times \mathbb{R}^{d}
$$


belongs to the product $\sigma$-algebra $\mathcal{F} \otimes \mathcal{B}\left(\mathbb{R}^{d}\right)$. In this case, $X$ is said to be a random set. In the same way the $\mathcal{H}$-measurability of $X$ with respect to a sub$\sigma$-algebra $\mathcal{H}$ of $\mathcal{F}$ is defined. The random set $X$ is said to be closed (convex, open) if $X(\omega)$ is a closed (convex, open) set for almost all $\omega$.

Definition 9.1 An $\mathcal{F}$-measurable random element $\xi$ in $\mathbb{R}^{d}$ such that $\xi(\omega) \in$ $X(\omega)$ for almost all $\omega \in \Omega$ is said to be an $\mathcal{F}$-measurable selection (selection in short) of $X, \mathcal{L}^{0}(X, \mathcal{F})$ denotes the family of all $\mathcal{F}$-measurable selections of $X$, and $\mathcal{L}^{p}(X, \mathcal{F})$ is the family of $p$-integrable ones.

It is known that an a.s. non-empty random set has at least one selection, see [21, Th. 4.4]. Let $\mathcal{H}$ be a sub- $\sigma$-algebra of $\mathcal{F}$.

Definition 9.2 A family $\Xi \subset \mathcal{L}^{0}\left(\mathbb{R}^{d}, \mathcal{F}\right)$ is said to be infinitely $\mathcal{H}$-decomposable if $\sum_{n} \xi_{n} \mathbf{1}_{A_{n}} \in \Xi$ for all sequences $\left(\xi_{n}\right)_{n \geq 1}$ from $\Xi$ and all $\mathcal{H}$-measurable partitions $\left(A_{n}\right)_{n \geq 1}$ of $\Omega ; \Xi$ is $\mathcal{H}$-decomposable if this holds for finite partitions.

The decomposable subsets of $\mathcal{L}^{0}\left(\mathbb{R}^{d}, \mathcal{F}\right)$ are called stable and infinitely decomposable ones are called $\sigma$-stable in [6]. The following result for $\mathcal{H}=\mathcal{F}$ is well known in case $p=1[22$, where the decomposability concept was first introduced; see also [28, Th. 2.1.10] for $\mathcal{H}=\mathcal{F}$, and [24, Prop. 5.4.3] for $p=0$.

Theorem 9.1 (see [26, Th. 2.4] and [28, Th. 2.1.10]) Let $\Xi$ be a nonempty subset of $\mathcal{L}^{p}\left(\mathbb{R}^{d}, \mathcal{F}\right)$ for $p=0$ or $p \in[1, \infty]$. Then

$$
\Xi \cap \mathcal{L}^{p}\left(\mathbb{R}^{d}, \mathcal{H}\right)=\mathcal{L}^{p}(X, \mathcal{H})
$$

for an $\mathcal{H}$-measurable random closed set $X$ if and only if $\Xi$ is $\mathcal{H}$-decomposable and closed.

For $A_{1}, A_{2} \subset \mathbb{R}^{d}$, define their elementwise (Minkowski) sum as

$$
A_{1}+A_{2}=\left\{x_{1}+x_{2}: x_{1} \in A_{1}, x_{2} \in A_{2}\right\} \text {. }
$$

The same definition applies to the sum of subsets of $\mathcal{L}^{0}\left(\mathbb{R}^{d}, \mathcal{F}\right)$. The set of pairwise differences of points from $A_{1}$ and $A_{2}$ is obtained as $A_{1}+\left(-A_{2}\right)$, or shortly $A_{1}-A_{2}$, where $-A_{2}=\left\{-x: x \in A_{2}\right\}$ is the centrally symmetric variant of $A_{2}$. For the sum $A+\{x\}$ of a set and a singleton we write shortly $A+x$. Note that the sum of two closed sets is not necessarily closed, unless at least one of the closed summands is compact. The following result differs from [28, Th. 1.3.25] in considering the possibly non-closed sum of two random closed sets.

Lemma 9.1 Let $X$ and $Y$ be two random sets. Then $\mathcal{L}^{0}(X, \mathcal{F})+\mathcal{L}^{0}(Y, \mathcal{F})=$ $\mathcal{L}^{0}(X+Y, \mathcal{F})$. If both $X$ and $Y$ are random closed sets, then $X+Y$ is measurable. 
Proof It is trivial that $\mathcal{L}^{0}(X, \mathcal{F})+\mathcal{L}^{0}(Y, \mathcal{F}) \subset \mathcal{L}^{0}(X+Y, \mathcal{F})$. To prove the reverse inclusion, consider $\xi \in \mathcal{L}^{0}(X+Y, \mathcal{F})$. Since $X$ and $Y$ are $\mathcal{F}$-measurable, the measurable selection theorem [24, Th. 5.4.1] yields that there exist $\mathcal{F}$ measurable selections $\xi^{\prime} \in \mathcal{L}^{0}(X, \mathcal{F})$ and $\xi^{\prime \prime} \in \mathcal{L}^{0}(Y, \mathcal{F})$ such that $\xi=\xi^{\prime}+\xi^{\prime \prime}$.

Now assume that $X$ and $Y$ are closed and consider their Castaing representations (see [28, Def. 1.3.6]) $X(\omega)=\operatorname{cl}\left\{\xi_{i}^{\prime}(\omega), i \geq 1\right\}$ and $Y(\omega)=\operatorname{cl}\left\{\xi_{i}^{\prime \prime}(\omega), i \geq\right.$ 1\}. The measurability of $X+Y$ follows from the representation

$$
\begin{aligned}
& \operatorname{Gr}(X+Y) \\
& =\bigcup_{k \geq 1} \bigcap_{m \geq 1} \bigcup_{i, j \geq 1}\left\{(\omega, x):\left\|x-\xi_{i}^{\prime}(\omega)-\xi_{j}^{\prime \prime}(\omega)\right\| \leq \frac{1}{m},\left\|\xi_{i}^{\prime}(\omega)\right\| \leq k\right\} .
\end{aligned}
$$

Indeed, if $(\omega, x) \in \operatorname{Gr}\left(X_{1}+X_{2}\right)$, then $x=a+b$ for $a \in X_{1}(\omega)$ and $b \in X_{2}(\omega)$. Let $k \geq 1$ such that $\|a\|+1 \leq k$. Since $a \in X_{1}$, there exists a subsequence $\left(\xi_{n_{l}}^{\prime}\right)_{l \geq 1}$ such that $\xi_{n_{l}}^{\prime}(\omega) \rightarrow a$. We may assume without loss of generality that $\left\|\xi_{n_{l}}^{\prime}(\omega)\right\| \leq k$. Similarly, $\xi_{n_{l}}^{\prime \prime}(\omega) \rightarrow b$. Therefore, if $m>0$, then $\| x-\xi_{i}^{\prime}(\omega)-$ $\xi_{j}^{\prime \prime}(\omega) \| \leq \frac{1}{m}$ and $\left\|\xi_{i}^{\prime}(\omega)\right\| \leq k$ for some $i, j$.

Acknowledgements IM was supported by the Swiss National Science Foundation Grant 200021-153597.

EM thanks the program Investissements d'Avenir from the French foundation ANR which supports the Bachelier colloquium, Metabief, France.

\section{References}

1. Acciaio, B., Penner, I.: Dynamic risk measures. In: Advanced mathematical methods for finance, pp. 1-34. Springer, Heidelberg (2011)

2. Cascos, I., Molchanov, I.: Multivariate risks and depth-trimmed regions. Finan. Stoch. 11, 373-397 (2007)

3. Çetin, U., Jarrow, R.A., Protter, P.: Liquidity risk and arbitrage pricing theory. Finance Stoch. 8(3), 311-341 (2004). DOI 10.1007/s00780-004-0123-x. URL http://dx.doi.org/10.1007/s00780-004-0123-x

4. Cerný, A., Hodges, S.: The theory of good-deal pricing in incomplete markets. In: H. Geman, M. D., S. Pliska, T. Vorst (eds.) Mathematical Finance. Bachelier Congress 2000, pp. 175-202. Springer, Berlin (2001)

5. Cheridito, P., Kupper, M., Tangpi, L.: Duality formulas for robust pricing and hedging in discrete time. SIAM J. Financial Math. 8, 738-765 (2017)

6. Cheridito, P., Kupper, M., Vogelpoth, N.: Conditional analysis on $\mathbb{R}^{d}$. In: A. Hamel, F. Heyde, A. Löhne, B. Rudloff, C. Schrage (eds.) Set Optimization and Applications in Finance - The State of the Art. Springer, Heidelberg (2015)

7. Cherny, A.S.: Pricing and hedging European options with discrete-time coherent risk. Finance Stoch. 11, 537-569 (2007)

8. Cherny, A.S.: Pricing with coherent risk. Theor. Probab. Appl. 52, 389-415 (2008)

9. Cherny, A.S., Grigoriev, P.G.: Dilatation monotone risk measures are law invariant. Finance Stoch. 11, 291-298 (2007)

10. Cherny 506-540 (2007)

11. Cochrane, J.H., Saá-Requejo, J.: Beyond arbitrage: Good deal asset price bounds in incomplete markets. J. of Political Econ. 108, 79-119 (2000)

12. Delbaen, F.: Monetary Utility Functions. Osaka University Press, Osaka (2012) 
13. Feinstein, Z., Rudloff, B.: A comparison of techniques for dymanic multivariate risk measures. In: A. Hamel, F. Heyde, A. Löhne, B. Rudloff, C. Schrage (eds.) Set Optimization and Applications in Finance - The State of the Art. Springer, Berlin (2015)

14. Feinstein, Z., Rudloff, B.: Multi-portfolio time consistency for set-valued convex and coherent risk measures. Finan. Stoch. 19, 67-107 (2015)

15. Filipović, D., Kupper, M., Vogelpoth, N.: Separation and duality in locally $L^{0}$-convex modules. J. Funct. Anal. 256, 3996-4029 (2009)

16. Filipović, D., Kupper, M., Vogelpoth, N.: Approaches to conditional risk. SIAM J. Financial Math. 3, 402-432 (2012)

17. Föllmer, H., Schied, A.: Stochastic Finance. An Introduction in Discrete Time, 2 edn. De Gruyter, Berlin (2004)

18. Grigoriev, P.G.: On low dimensional case in the fundamental asset pricing theorem with transaction costs. Statist. Decisions 23, 33-48 (2005)

19. Hamel, A.H., Heyde, F.: Duality for set-valued measures of risk. SIAM J. Finan. Engineering 1, 66-95 (2010)

20. Hamel, A.H., Rudloff, B., Yankova, M.: Set-valued average value at risk and its computation. Math. Finan. Economics 7, 229-246 (2013)

21. Hess, C.: Set-valued integration and set-valued probability theory: An overview. In: E. Pap (ed.) Handbook of Measure Theory, chap. 14, pp. 617-673. Elsevier (2002)

22. Hiai, F., Umegaki, H.: Integrals, conditional expectations, and martingales of multivalued functions. J. Multiv. Anal. 7, 149-182 (1977)

23. Kabanov, Y., Rásonyi, M., Stricker, C.: No-arbitrage criteria for financial markets with efficient friction. Finance Stoch. 6(3), 371-382 (2002). DOI 10.1007/s007800100062. URL http://dx.doi .org/10.1007/s007800100062

24. Kabanov, Y.M., Safarian, M.: Markets with Transaction Costs. Mathematical Theory. Springer, Berlin (2009)

25. Lépinette, E.: Robust no arbitrage condition for continuous-time models with transaction costs. In: M. Kijima, C. Hara, Y. Muromachi, H. Nakaoka, K. Nishide (eds.) Recent Advances in Financial Engineering, Proceedings of the Kier-Tmu international workshop on financial engineering, pp. 69-82. World Scientific, Singapore (2010)

26. Lépinette, E., Molchanov, I.: Conditional cores and conditional convex hulls of random sets. J. Math. Anal. Appl. 478, 368-392 (2019)

27. Löhne, A., Rudloff, B.: An algorithm for calculating the set of superhedging portfolios and strategies in markets with transaction costs. Int. J. Theor. Appl. Finance 17, 1450012 (33 pages) (2014)

28. Molchanov, I.: Theory of Random Sets, 2nd edn. Springer, London (2017)

29. Molchanov, I., Cascos, I.: Multivariate risk measures: a constructive approach based on selections. Math. Finance 26, 867-900 (2016)

30. Pennanen, T., Penner, I.: Hedging of claims with physical delivery under convex transaction costs. SIAM J. Finan. Engineering 1, 158-178 (2010)

31. Rásonyi, M.: New methods in the arbitrage theory in financial markets with transaction costs. In: C. Donati-Martin, M. Émery, A. Rouault, C. Stricker (eds.) Séminaire de Probabilités XLI, Lect. Notes Math., vol. 1934, pp. 455-462. Springer, Berlin (2008)

32. Schachermayer, W.: The fundamental theorem of asset pricing under proportional transaction costs in finite discrete time. Math. Finance 14, 19-48 (2004)

33. Vogelpoth, N.: $L^{0}$-convex analysis and conditional risk measures. Ph.D. thesis, Universität Wien (2009)

34. Willesson, M.: What is and what is not regulatory arbitrage? A review and syntheses. In: P.A. Chesini G. Giaretta E. (ed.) Financial Markets, SME Financing and Emerging Economies. Palgrave Macmillan, Cham (2017) 